\title{
LA MEDIDA DE LA PERCEPCIÓN SOCIAL DEL MEDIO AMBIENTE \\ Una revisión de las aportaciones realizadas por la sociología*
}

\author{
J. DAVID TÁBARA \\ Universidad de Pompeu Fabra. Barcelona
}

\section{PALABRAS CLAVE ADICIONALES}

Cambio de paradigma cultural, Evaluación

Ambiental Integrada (EAI), Sociología ambiental.

\section{ADDITIONAL KEYWORDS}

Cultural Paradigm Change, Integrated Environmental Assessment (IEA), Environmental Sociology.

RESUMEN. El presente artículo revisa las metodologias y los resultados de medición de la percepción ambiental por parte de la sociología. En primer lugar, examina cómo esta disciplina ha resuelto en el ámbito internacional un conjunto de cuestiones entrelazadas respecto al qué medir, quién medir, cómo medir y para qué medir. En segundo lugar, hace un repaso de las aportaciones realizadas en España hasta el momento, detectándose cinco características que, en opinión del autor, suponen importantes escollos para el pleno desarrollo de este campo de investigación: (a) fragmentación e incomparabilidad; (b) falta de sintesis y debilidad teórica; (c) preferencia cuantitativa; (d) irregularidad; y (e) falta de integración. Finalmente, se enmarcan los principales argumentos expuestos dentro de una discusión más amplia respecto a las tensiones que padecen actualmente las ciencias sociales ambientales en general. El objetivo es contribuir a la consolidación de este campo de investigación y estimar algunos criterios que ayuden a evaluar la relevancia de lo que se ha medido.

\footnotetext{
* El origen de este artículo se encuentra en la ponencia presentada en el seminario "Análisis de la conciencia ambiental en la opinión pública", celebrado el 12 de abril del 2000 en el Instituto de Estudios Sociales de Andalucia del Consejo Superior de Investigaciones Cientificas. Agradezco a Eduardo Moyano su invitación a participar en el encuentro y a Vicente Carabias la coordinación del mismo.

E-mail: jdtabara@terra.es
}

Revista Internacional de Sociología (RIS)

Tercera Época, n 28 , Enero-Abril, 2001, pp. 127-171. 
RIS

RETISTA INTERNACIONaL DE SOCIOLOGIA

N" 28. Enerv-Abril. 200

SUMMARY. The present article reviews the social methodologies used by sociology as well as some of the main results obtained in the study of environmental perception. First, it examines how this discipline has answered a set of interwoven questions regarding to what to measure, whom to measure, how to measure and what to measure for. Next, it reviews the contributions provided by some Spanish researchers in this field, which according to the author, still suffers from: (a) fragmentation and lack of comparability; (b) lack of synthesis and theoretical debility; (c) preference for quantitative designs; (d) irregularity; and (e) lack of integration. Finally, the conclusion is framed within a broader discussion related to the main debates and tensions which affect current social environmental sciences. This review aims not only to contribute to the consolidation of this line of research within sociology but to provide some criteria to evaluate the relevance of what has been measured.

\section{INTRODUCCIÓN}

En un artículo casi olvidado por las ciencias sociales ambientales, David Lowenthal (1987) hacía un repaso de lo que en los años sesenta emergía como un prometedor campo de investigación: el estudio de la percepción social del medio ambiente. Siendo este autor uno de los maestros en esta materia dentro de la geografia, Lowenthal comentaba que su intención era aportar una explicación de cómo "la gente da sentido al mundo que le rodea" (p. 338), un propósito que contrastaba con el clima realista imperante en su época, y que se centraba más en dar un listado de los elementos "objetivos" que condicionan la percepción ambiental, que en mostrar que tales percepciones están mediatizadas por "experiencias y preconcepciones, coloreadas por los gustos y las preferencias, remoldeadas por la memoria y la amnesia, que guían nuestros juicios y acciones ambientales" (p. 338). Los estudios de percepción ambiental prometían mucho, y con ellos, también crecían las expectativas de encontrar nuevos recursos y nuevos usos para más investigaciones. No obstante, y en un atrevido balance de quince años de esfuerzos previos en esta materia, este mismo autor concluía diciendo que:

"este nuevo campo de investigación ha prestado una atención insuficiente por lo que se refiere a la teoría y a la síntesis. En estos 15 años se ha visto muy poco progreso fundamental. La mayor parte de esta investigación parece ser estrecha de campo, sin vida en el tono y trivial en sus resultados. Los avances interesantes se pueden encontrar en un buen número de las periferias, pero el centro parece vacío de espíritu" (Lowenthal, 1987: 337).

Los avisos de Lowenthal deberían actuar de revulsivo para evitar caer en pasados errores cometidos por otras disciplinas y al mismo tiempo deberían incitar a plantearse un conjunto de cuestiones relevantes cuando nos proponemos medir la percepción ambiental. Dado que éste no es el lugar para hacer una 
extensa revisión de las respuestas ofrecidas por la psicología, la geografía o la historia - todas ellas con una vasta literatura en el área de la investigación de la percepción social del medio ambiente-, en este artículo me limitaré, en primer lugar, a revisar algunas de las aportaciones realizadas por la sociología en el ámbito internacional. En particular, se trata de ver cómo se han resuelto determinadas espinosas decisiones estratégicas de carácter metodológico relativas al qué medir, a quién medir, cómo medir, y, ante todo, para qué medir. En segundo lugar, comentaré, brevemente, algunos de los trabajos realizados en España hasta la fecha en materia de medición de la percepción ambiental. $\mathrm{Y}$ finalmente, concentraré mi atención en algunos de los criterios que podrían tenerse en cuenta para poder evaluar lo que se ha medido. De este modo, el presente artículo no se interesa tanto por efectuar un análisis comparativo y detallado de los resultados obtenidos a través de estos trabajos, como por dar a conocer sus objetos de estudio, observar las opciones escogidas, seleccionar algunas conclusiones más destacadas y evaluar los procedimientos metodológicos elegidos en cada caso.

\section{APORTACIONES DE LA SOCIOLOGÍA}

Cualquier investigador que se enfrente al estudio y al análisis de la percepción social del medio ambiente deberá responder de manera inevitable a un conjunto de preguntas que condicionarán decisivamente tanto la estrategia, como el contenido de los resultados obtenidos en sus pesquisas. Se trata de cuestiones cuyas respuestas están íntimamente ligadas tanto a la teoría social, como al contexto donde se aplican y que, por tanto, no pueden obtenerse sin aplicar un cierto grado de discrecionalidad. De hecho, la misma selección de estas preguntas, y no otras, es en sí misma un acto discrecional.

\section{¿Qué medir?}

La mayor parte de los objetos de investigación de los trabajos llevados a cabo por la sociología en el ámbito de la percepción ambiental se puede agrupar en dos grandes campos. En primer lugar, ha habido una notable actividad internacional dedicada al estudio de la percepción de determinados problemas ambientales $y$ de las interacciones sociales que tales problemas provocan. En particular, se han estudiado el nivel de preocupación pública por la problemática ambiental, el grado de información que disponen las poblaciones analizadas y las fuentes de información que más utilizan, así como las disponibilidades a actuar o las medidas políticas que se perciben como más favorables para enfrentarse a tales problemas ambientales. En este sentido, también se ha ahondado en los factores estructurales que explican o influyen en los contrastes de las diversas 
R I S

REIISTA INTERNACIONAL DE SOCIOLOGIA

No 28. Enero--thril. 2001

J. DAVID TÁBARA

percepciones, como son el nivel educativo, el lugar de residencia, la edad, el sexo o las diferencias étnicas. En este caso, tales factores estructurales se toman como variables independientes para explicar la percepción ambiental por parte de la población.

En segundo lugar, la investigación internacional ha analizado el contenido y el proceso de cambio de los diferentes paradigmas culturales que explicarían una actitud más favorable a la protección y a la mejora del medio ambiente. Estos nuevos paradigmas emergentes han adoptado en la literatura social diferentes denominaciones, como son los de "ecológico", "post-material", "alternativo" o "sostenible", todos ellos con elementos comunes, pero también con matices y contrastes diferenciadores notables. Si bien es cierto que se tratan de dos áreas distintas: una mucho más descriptiva y la otra mucho más teórica, no debe entenderse que las investigaciones entre ambos campos están desligadas, dado que existen múltiples conexiones. En numerosos casos, la investigación sobre la percepción de problemas ambientales, y en concreto sobre la estimación por parte de la población de la gravedad de los problemas que afectan al medio ambiente, el uso de energía nuclear o la mayor propensión a la protección ambiental en detrimento de la defensa del crecimiento económico, se ha utilizado para justificar empíricamente el cambio cultural hacia estos nuevos paradigmas emergentes.

\section{La percepción de los problemas ambientales y sus interacciones sociales}

En este área, se ha seleccionado una serie de temas de investigación bastante diversos y que podemos englobar genéricamente bajo la denominación interacciones o respuestas sociales, entre los cuales destacan los siguientes:

a) El estudio del nivel de preocupación y la estimación de la gravedad o importancia de los diferentes problemas y riesgos ambientales.

b) El examen del grado de información y de conocimiento de la problemática ambiental; las fuentes de información más utilizadas; y la evaluación pública de tales fuentes.

c) La exploración de los comportamientos ambientales; el apoyo ciudadano a la protección del medio ambiente; y las preferencias y disponibilidades de la población a llevar a cabo o a aceptar, medidas políticas de carácter ambiental.

d) El análisis de contrastes que aparecen en los anteriores temas en función de diferencias observables en las características socioeconómicas de los individuos de la muestra correspondiente.

Dado que la mayoría de los trabajos que abordan la percepción social de los problemas ambientales y sus interacciones tiende a examinar el conjunto de tales temas a la vez, a continuación únicamente se comenta la evolución de los estudios y autores más destacados en este terreno sin detallar de manera pormenorizada y separada cada uno de los elementos tratados en cada trabajo. 


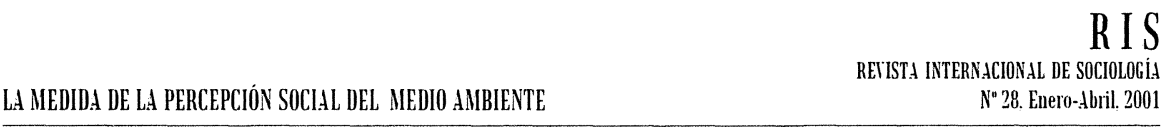

Aunque existe un número limitado de trabajos anteriores a la década de los setenta, podemos afirmar que fue principalmente a partir de esa fecha y de la labor realizada por los sociólogos estadounidenses Frederick H. Buttel, Riley Dunlap y sus colaboradores respectivos, cuando se marca el inicio de la investigación empírica en sociología de las percepciones ambientales y sus interacciones sociales. La mayoría de estos estudios consistió en sondeos de opinión dirigidos a grandes poblaciones, cuyas variables fueron operacionalizadas mediante escalas Likert de 4 a 10 valores. Por ejemplo, uno de estos primeros trabajos orientados a la estimación del nivel de relevancia pública de los problemas que afectan al medio ambiente fue llevado a cabo por Buttel, Martinson y Flinn (1975). En aquella investigación demostraron que los ciudadanos de los Estados Unidos ordenaban los distintos problemas del medio ambiente en función de dos dimensiones básicas, calificadas como "utilitaria", por un lado, y "conservacionista", por otro. Según sus resultados, la valoración de la importancia de los problemas ambientales por parte de la mayoría del público estadounidense se hacía sobre la base de criterios "utilitarios", por lo que los problemas ambientales más urgentes eran aquéllos que afectaban directamente a las personas - tales como los de contaminación del agua y aire-; tales resultados señalaban también que los factores estructurales que explicaban las diferencias en la estimación de la gravedad eran el nivel educativo y el lugar de residencia. El mismo Buttel y sus colaboradores abordaron ya entonces, en otro sondeo cuantitativo, pionero la que sería una perenne cuestión en este área de trabajo ẏ que gira en torno al dilema entre crecimiento económico y protección ambiental (Buttel y Flinn, 1976).

Asimismo, y en este mismo conjunto de temas de investigación, el ingente trabajo cuantitativo llevado a cabo por Dunlap durante más de dos décadas le permitió asegurar lo siguiente: que en los Estados Unidos la preocupación por los problemas ambientales había crecido a finales de los años sesenta, hasta llegar a un máximo en el año 1970, momento de la primera celebración del Día de la Tierra; que a partir de entonces, y durante los primeros años siguientes, tal preocupación descendió de modo muy importante y de manera más gradual al final de esa década; y que, en la década de los ochenta, la preocupación ambiental en los Estados Unidos volvió a ascender de modo muy considerable y regular hasta llegar al veinte aniversario del Día de la Tierra, momento en que la preocupación por el medio ambiente llegó a su momento más álgido (Dunlap, 1991a y 1991b; Dunlap y Dillman, 1976; Dunlap y Scarce, 1991; Dunlap y Van Liere, 1977; Dunlap; Van Liere y Dillman, 1979)'.

\footnotetext{
${ }^{1}$ En Europa, los datos al respecto son mucho más escasos y varian mucho según los países, aunque por ejemplo, según las estadísticas facilitadas por Eurostat, ya en el año 1992 el $85 \%$ de los ciudadanos de la Unión Europea calificaba como urgentes e inmediatos a los problemas ambientales.
} 
RIS

RETISTA INTERNACIONAL DE SOCIOLOGIA

N" 28, Enero-Abril. 2001

J. DATIDTÁBARA

El estudio de las fuentes de información y del grado de conocimiento ambiental ha recibido menor atención por parte de los principales sociólogos ambientales, aunque en muchos casos estos temas también han sido incluidos en sus trabajos. Por ejemplo, Dunlap (1998) analiza los temas de información y conocimiento sobre el cambio climático en seis países, concluyendo que se trata de un problema que se confunde con otros problemas y que se comprende poco. A la misma conclusión llegan otros autores como Bostrom et al. (1994) y Read et al. (1994), aunque la metodología utilizada, también de carácter cuantitativo, es algo dudosa dado lo reducido y lo poco representativo de las muestras que seleccionaron. Por otro lado, Tábara (1995) analizó la correlación entre información y preocupación ambientales a partir de una muestra de 200 individuos, no encontrando ninguna relación significativa y proponiendo la hipótesis de que tal relación quizás sea incluso inversa, pero asimétrica. Es decir, a mayor información, menor preocupación (especialmente a partir de un punto de sobreinformación), aunque a mayor preocupación mayor demanda de información.

En el campo de la información ambiental también ha habido una considerable labor destinada al examen de los medios de comunicación, valorándose entre otros aspectos el grado de utilización, fiabilidad, credibilidad e influencia. En este área, los enfoques cualitativos han disfrutado de un mayor protagonismo. De especial relevancia metodológica es la aportación de Lacey y Longman (1997) en la que comparan los resultados de opinión pública ambiental durante cinco años en el Reino Unido producidos a través de la agencia MORI, con los obtenidos mediante un vaciado de contenido de periódicos nacionales a través de la codificación de palabras clave, produciéndose una correlación sorprendentemente alta entre ambos $(\mathrm{R}=0,79$ al 0.001). En Estados Unidos, resultados igualmente correlacionados entre el vaciado de contenido de periódicos y los datos de sondeos obtenidos por Dunlap, se dan en Hoffman (1996). Si bien las relaciones de causalidad entre opinión pública y medios de comunicación son discutibles, y nunca unidireccionales, Bell (1994) argumenta que los medios de comunicación sólo contribuyen a la mejora de la comprensión de problemas ambientales complejos, como el cambio climático, en tanto que las audiencias respectivas ya conocen algo por su cuenta sobre estos temas. El examen de la información y medios de comunicación en temas ambientales a través de sondeos de opinión ya fue abordado, entre otros, por Ostman y Parker a mediados de los ochenta (1986-87; Tábara, 1993); en los noventa, destaca la aportación de Chapman et al. (1997) en donde un mismo cuestionario con preguntas relativas a los medios de comunicación y el medio ambiente se utilizó simultáneamente en la India y en el Reino Unido.

En relación con las actividades ambientales más comunes o las medidas que deberían tomarse, los temas elegidos de modo más usual por los trabajos de percepción en este terreno, pueden clasificarse en dos subáreas. En la primera 
se incluirían los estudios sobre opinión respecto a lo que los demás deberían hacer (e.g.: el gobierno, las empresas o los otros ciudadanos); en la segunda, las acciones de lo que uno mismo estaría dispuesto a llevar a cabo o aceptar, para "resolver" los problemas ambientales ${ }^{2}$. En relación con la primera, destacan las preguntas en torno la valoración de si el gasto público en materia de conservación y prevención ambiental es suficiente o no, asi es necesario crear una legislación ambiental más severa, o a si se debería fomentar mucho más la investigación, la información o la educación del público en esta materia. En este campo, también se tratan cuáles son los principales responsables de la crisis ambiental, tanto de causar los problemas, como de actuar para enfrentarse a ellos. Respecto al segundo subárea se pregunta sobre qué acciones ambientales el encuestado ya realiza o estaría más dispuesto a realizar en su vida cotidiana, y en particular si estaría dispuesto a participar en las campañas de reciclado de materiales, en formar parte o dar apoyo a grupos ambientalistas o en cursar denunciăs ante delitos o infracciones ambientales. En este sentido, algunos organismos internacionales, como la OCDE y la Unión Europea a través del Eurobarómetro ${ }^{3}$, también aportan datos comparables y relevantes sobre acciones y preferencias ambientales, entre las cuales destaca la tradicional y omnipresente pregunta relativa al dilema entre crecimiento económico y protección ambiental o sobre en qué medida se estaría dispuesto a aceptar una reducción del crecimiento económico si esto garantizase una mayor protección ambiental. También es frecuente pedir a los entrevistados su opinión sobre la disponibilidad a aceptar un incremento de impuestos o de precios para asegurar la mejora ambiental, de modo paralelo a lo que ocurre en economía, en donde la evaluación contingente de los bienes y servicios ambientales se basa en la aplicación de la metodología de sondeos cuantitativos para la estimación de las disponibilidades a pagar o a aceptar determinados valores monetarios ${ }^{4}$.

\footnotetext{
${ }^{2}$ Esta distinción entre lo que uno hace o está dispuesto a hacer y lo que se espera que hagan los demás es útil, puesto que permite observar aquellas áreas de acción ambiental donde los individuos mantienen un sentimiento de competencia política superior - donde se percibe que los elementos de agencia superan los de estructura- y donde las posiciones del "gorrón" de Mancur Olson (free-rider) pueden estar más presentes (Tábara, 1995).

${ }^{3}$ Otros tres trabajos que contienen datos de sondeos comparativos realizados a nivel internacional en materia de actividades ambientales son los de ODCE (1991), Skrentny (1993), Weber y Corrado (1993); y Worcester (1994); en particular, los trabajos de la ODCE y de Weber y Corrado incluyen resultados de estudios referidos a España - uno de los pocos casos en que tal comparación internacional de los resultados españoles es posible- a partir de una muestra realizada en el primer caso en 1988 a 1.017 individuos, y a 1.007 individuos en 1992, en el segundo.

+ Para un tratamiento más amplio del concepto de acción ambiental y datos recogidos mediante sondeos de opinión al respecto puede verse Tàbara (1999b), y para el caso de Canadá, Blake et al. (1997).
} 


\section{RIS}

Respecto al cuarto gran conjunto de temas de investigación, el de las características estructurales que predicen o explican la percepción ambiental, la mayor parte de los sondeos cuantitativos que analizan los grados preocupación o de información ambiental realizan análisis bivariantes y multivariantes diversos, realizando cruces con variables de tipo socioeconómico. A partir de ahí, y en tanto que se parta de muestras suficientemente grandes y se realicen los respectivos controles, es posible investigar si existen diferencias representativas entre las evaluaciones realizadas por las distintas subpoblaciones de la muestra o si aparecen factores que discriminen la percepción de determinados problemas. Entre los principales contrastes estudiados al respecto destacan los que aparecen entre hombres y mujeres, entre los diferentes grupos de edad, entre los residentes urbanos y los residentes no urbanos ${ }^{5}$, entre los diferentes niveles educativos, entre los distintos estratos socioeconómicos, entre los países o áreas pobres y ricas, entre diferentes áreas administrativas o políticas, entre los individuos expertos y el público no experto en general ${ }^{6}$, entre los que su profesión se encuentra en el centro o en la periferia de la economía competitiva de mercado, entre los que demuestran posiciones ideológicas políticamente distintas, entre los que defienden tendencias nacionalistas o no, y entre los que llevan a cabo distintas actividades de ocio y, en particular, realizan o no actividades recreativas al aire libre ${ }^{7}$. Igualmente, la mayoría de estudios que correlacionan variables de percepción o información con características socioeconómicas, también lo hacen a la hora de explicar las causas de la acción ambiental. De notable mención en este sentido, dado que la mayoría de trabajos en este campo sólo describen pero no explican, es el análisis de los 230 individuos de la muestra de Carlo Jaeger et al. (1993) que les llevó a defender que los condicionantes de la acción personal en materia de cambio climático son principalmente las redes sociales y las normas interpersonales.

También en esta línea, Dunlap y sus colaboradores se interesaron en profundizar en el conocimiento de las "bases sociales" de la preocupación ambiental. Así, darían origen a otro conjunto de trabajos que permitirían realizar un seguimiento de la variabilidad de estos sectores sociales en los Estados Unidos durante dos décadas. Sus resultados daban a conocer que tal base no

\footnotetext{
5 Por ejemplo, Lowe y Pinhey (1982) enfatizan el distinto modo de socialización y la permanencia de una visión mas instrumental de la naturaleza en las zonas rurales (Arcury, 1993).

${ }^{6}$ Así por ejemplo, ya en un trabajo encargado por las Naciones Unidas a la organización Harris, ya se observaba cómo los expertos tendían a valorar los problemas ambientales de modo distinto al público en general. Los elementos asociados a la energía y los residuos nucleares o los accidentes con las industrias químicas eran evaluados por el público como actividades de alto riesgo, mientras que los expertos les concedian menor importancia (Harris/UNEP, 1988).

${ }^{7}$ En este último caso la evidencia empírica era nula o relativamente débil (Van Liere y Noe, 1981); conclusión a la que llegó Geisler (1977).
} 
había variado significativamente y que en concreto, las subpoblaciones más preocupadas tendían a ser adultos-jóvenes, con mayor formación, votantes demócratas, con tendencias liberales, de zonas urbanas y con empleos fuera del sector primario (Dunlap, 1992a; Jones y Dunlap, 1992; Van Liere y Dunlap, 1980 y 1981). Paralelamente Buttel (1987) observaba que otras variables sociodemográficas, como la educación, la edad o, en menor medida, el lugar de residencia, permitían predecir la preocupación ambiental, aunque el mejor predictor en este sentido era la ideología política y más específicamente, el liberalismo, que aparecía como el factor determinante.

Estas discusiones formaban parte de un debate que se producía a ambos lados del Atlántico, a saber: el debate en torno a la cuestión de si el movimiento ambientalista constituía un movimiento de carácter elitista, un movimiento de radicalismo de clase media o si no era nada de eso, por lo que sus características sociales no se distinguían significativamente de las de otros movimientos sociales (Morrison y Dunlap, 1986). Por otro lado, Mohai (1985), desde la perspectiva de la psicología social, ya distinguió entre los activistas y los que dan apoyo al ambientalismo, expresando que en todo caso el posible elitismo sólo se podría dar en los primeros pero no en los segundos. El mismo Cotgrove (1980 y 1982) abordó estas cuestiones con respecto a si la preocupación por los problemas ambientales, el apoyo a medidas ambientales o la participación ambientalista son fenómenos propios de lo que Frank Parkin denominaba "radicalismo de clase media" americanos en tanto que:

"Existe poca evidencia que sugiera que la preocupación ambiental sea fuero exclusivo de un grupo social particular, [aunque...] los ambientalistas generalmente son más jóvenes, de izquierdas, trabajadores de empleos que están apartados del mercado, y con mayores ingresos que el individuo medio" (Cotgrove, 1982:135-136).

En todos estos estudios, la percepción de la gravedad, del conocimiento o de las acciones sociales respecto a los problemas ambientales ha sido tratada entendiendo el medio ambiente en su conjunto o bien, en un proceder más propio de la sociología ambiental, de manera más especifica, detallando a qué problemas en concreto se referían. Los problemas ambientales abordados de

\footnotetext{
${ }^{8}$ Por lo que se refiere a la posible defensa de transformaciones "radicales" hacia la sostenibilidad, hay muchos argumentos para creer más bien lo contrario, pues las clases medias tienden a tener más razones para ser conservadoras y a menudo muestran mayor aversión al cambio y al riesgo, lo cual queda demostrado por el hecho de que la inexistencia de clases medias en un país es entendido como frecuentemente un motivo de inestabilidad social.
} 
modo más frecuente han sido la contaminación del aire y del agua, los residuos, la escasez de energía, la sobrepoblación, la lluvia ácida y la pérdida de diversidad biológica. Más frecuentemente se observa el creciente interés por incorporar aspectos relativos al Cambio Ambiental Global (CAG), como el debilitamiento de la capa del ozono, la desertificación y deforestación o el cambio climático. Por ejemplo, Kempton et al. (1995) concentran buena parte de su atención al estudio del contenido y de las variaciones de los "modelos culturales" que afectan la apreciación del medio ambiente global, y en particular del cambio climático. Su aproximación, cualitativa, es original en cuanto realizaron un total de 43 entrevistas semi-estructuradas a partir de las cuales obtuvieron un conjunto de 149 afirmaciones representativas del conjunto de opiniones expresadas por los entrevistados, a las que fue posible asignar una medida porcentual de acuerdo-desacuerdo (escala likert de 5 valores) para cada una de ellas. Asimismo, los temas de la energía nuclear han sido objeto de notables producciones y es una de las áreas donde se dispone de las series temporales de datos más completas (Berk y Schulman, 1995; Czech, 1999; Dunlap, 1998; Rosa y Dunlap, 1994, y Tábara, 1996 y 2000a).

Por último, debe tenerse en cuenta que, a pesar de este importante trabajo científico, la mayor parte de los trabajos que actualmente se realizan para medir la percepción de los problemas ambientales en la opinión pública son, en realidad, muy descriptivos, y se resisten al análisis explicativo. De este modo, en realidad, muchos de ellos no superan la categoría de estudios de mercado, siendo a menudo llevados a cabo por consultorías por encargo de las Administraciones públicas, las cuales difícilmente se muestran interesadas en conectar los resultados obtenidos con la teoría social de modo que puedan ser comparados a nivel internacional. Ello supone, al margen de su coste económico (privado o público y que acostumbra a no ser nada despreciable), una pérdida de notables oportunidades para la consolidación de este campo de investigación en las ciencias sociales ambientales?

\section{Los cambios de paradigmas culturales}

Fue también a partir de los años setenta cuando empezaron a surgir un gran número de trabajos encaminados no sólo a medir la opinión, la información $\mathrm{u}$ otras respuestas sociales ante determinados procesos de cambio ambiental, sino también a evaluar si estas nuevas percepciones e interacciones atendían a

\footnotetext{
${ }^{9}$ Éste es el caso, entre otros muchos ejemplos, de los trabajos que han llevado a la elaboración anual de un "índice de concienciación ambiental" por parte del Departamento de Medio Ambiente de la Generalitat de Cataluña, que ignoró más de veinticinco años de investigación en este campo (Marketing Systems, 1996).
} 
un cambio cultural mucho más profundo. En cierto modo, puede concebirse el conjunto de este notable esfuerzo como el intento de proveer de un enfoque más macrosocial y de superar la falta de teoría y de síntesis que predominaba en los trabajos sobre percepción de los problemas ambientales y sus interacciones sociales hasta el momento.

Fueron precisamente Dunlap, Van Liere y Catton los primeros en sintetizar de manera operativa un conjunto de ocho dimensiones que identificaban como "Paradigma Social Dominante", de modo que podían ser relacionadas con el apoyo o el rechazo del público a la protección ambiental o al control de la contaminación (Dunlap, Catton y Van Liere, 1978 y 1984; Catton y Dunlap, 1978 y 1980). A partir de ahí, crearon una escala con doce dimensiones, que fue revisada y extendida a quince, y que ha sido utilizada considerablemente por la sociología ambiental (Grendstad, 1999). Estos autores incorporaron las críticas (entre otros) de Allan Schnaiberg, y cambiaron el nombre de "Paradigma Excepcionalista Humano" (aceptando que la especie humana sí era excepcional) por "Paradigma Exencionalista Humano" (PEH), para referirse de manera más específica a aquella visión cultural que cree que los seres humanos están exentos de las constricciones ecológicas. Esta dicotomía servía tanto para categorizar las distintas poblaciones que aparecían en sus análisis, como para saber cuál era el denominador común de los sociólogos ambientales que les permite distinguirse del resto de sociólogos (incluso de aquéllos que tratan temas de medio ambiente), siendo estos últimos los que ignoran completamente la interdependencia entre el ambiente biofisico y la sociedad en sus interpretaciones (Dunlap y W. R., 1983 y 1994b). Para estos autores (Catton y Dunlap, 1980; Dunlap y Catton, 1994b) los valores y creencias que caracterizan el PEH son los siguientes ${ }^{10}$ :

1. Los humanos disponen de un patrimonio cultural añadido (y distinto) a su patrimonio genético, por los que son diferentes a cualquier otra especie animal.

2. Los factores sociales y humanos (incluyendo la tecnología) son los principales determinantes de los asuntos humanos.

3. Lo crucial en los asuntos humanos son los contextos sociales y culturales, siendo irrelevante en gran medida el ambiente biofísico.

4. La cultura es acumulativa, por lo que el progreso tecnológico y social puede continuar indefinidamente, lo que en última instancia convierte todos los problemas ambientales en solucionables.

${ }^{10}$ Según Dunlap y Catton, el Paradigma Exencionalista Humano (PEH) guarda un gran paralelismo con una perspectiva más general y predominante que denominan "Visión Dominante Occidental". Para su comparación respectiva puede verse Dunlap y Catton (1994b: 16). 
Tales valores contrastaban con los valores y creencias del "Nuevo Paradigma Ecológico", que se caracterizaban por entender que:

1. Si bien la especie humana es fundamentalmente diferente (excepcional), es una de las muchas especies del ecosistema con las que mantiene relaciones de interdependencia.

2. Los asuntos humanos no sólo dependen de factores sociales y culturales sino también de sus interdependencias con la naturaleza, por lo que se dan muchas consecuencias no intencionadas.

3. Los humanos viven y dependen de un ambiente biofisico finito, que les impone fuertes constricciones fisicas y biológicas.

4. No obstante, y a pesar de que la inventiva humana, o los poderes que se derivan de ella, haga parecer que los límites de la capacidad de carga pueden traspasarse, las leyes ambientales no pueden ignorarse.

Y a partir de ahí, revisaron sus propias aportaciones para reformular lo que ahora denominan "Escala NPE Revisada" (Dunlap et al., 2000). De esta nueva versión, especificaron las quince dimensiones que ahora se usan para medir la adhesión al PEH o al NPE, agrupables en cuatro categorías y que se muestran en el esquema adjunto.

Por otro lado, el trabajo empírico de Stephen Cotgrove y Andrew Duff también se interesó en observar cuáles eran las creencias y los valores centrales del "Paradigma Social Dominante" y que prevalecían en sociedades industriales como Gran Bretaña. Al tiempo se trataba de ver en qué medida se contraponían con los valores que mantenían los sectores de población más favorables a los planteamientos ecologistas, y que entraban dentro de lo que denominaba como "Nuevo Paradigma Ambiental". Cotgrove distinguían un grupo, al que llamaba "industrialista" o "cornucopiano" (haciendo una clara alusión a los que creen en el cuerno de la abundancia), formado por individuos que defendían a ultranza el crecimiento económico, mostraban una posición instrumental y de dominación sobre naturaleza y creían en la existencia de recursos ilimitados; "ambientalista", "utópico" o "catastrofista" según los casos. El conflicto que se produce entre ambos grupos, que "habitan mundos diferentes", no es sobre el medio ambiente en sentido estricto, sino que consiste más bien en un enfrentamiento entre "cosmologías", entre visiones del mundo:

"la clave de lo diferente en los modos en que los ambientalistas y los industrialistas -catastrofistas y cornucopianos- ven a su ambiente debe hallarse en los contrastados patrones de creencias y valores (....); lo que está en juego no es solamente la contaminación de un río, o las vidas de unas focas, sino, como hemos visto, las visiones en conflicto sobre el orden moral y social" (Cotgrove, 1982: 26 y 85).

Y esto sucede porque en realidad, 
"Aquello que nos están diciendo los ecologistas es que la sociedad que tenemos está mal; que el modo como nos comportamos está en contra de 'La Naturaleza'; que nuestros hijos sufrirán, y que el tiempo se está agotando (...) quieren un tipo de sociedad diferente. $Y$ usan el medio ambiente como una palanca para intentar dar lugar a los cambios que ellos quieren" (p. 33-34).

Si bien tanto los trabajos de Dunlap y sus colegas, como de Cotgrove se centraban solamente en un solo país (Estados Unidos o Gran Bretaña), Milbrath (1984) fue el primero en abordar más en profundidad el estudio a la vez empírico (cuantitativo) y teórico sobre el posible cambio de paradigma cultural desde una perspectiva internacional. Su trabajo de campo comprendía dos encuestas, la primera en 1980 y después en 1982, realizadas en los Estados Unidos, en Inglaterra y en la antigua Alemania Federal. Este autor incorporó y retocó (en buena parte, bajo una perspectiva funcionalista y evolucionista) el enfoque de los anteriores autores en tanto que argumentaba que todas las sociedades disponen de un paradigma social dominante. Milbrath entendía por paradigma:

"la estructura dominante de creencias que organiza el modo que las personas perciben e interpretan el funcionamiento del mundo a su alrededor (...). Un paradigma social contiene la información de supervivencia [survival information] necesaria para el mantenimiento de una cultura. Es el resultado de generaciones de aprendizaje social, en donde aquellas creencias y valores disfuncionales son desechadas a favor de las más acordes con la supervivencia colectiva" (Milbrath, 1984: 7).

Partiendo de este enfoque teórico, Milbrath distinguió - y aplicó a su análisis empírico- entre aquellos colectivos o subpoblaciones que formaban parte de la "retaguardia" de la sociedad y defendían el viejo "Paradigma Social Dominante", y los que constituían la "vanguardia" de una nueva sociedad, es decir los que luchaban por extender un "Nuevo Paradigma Ambiental". Los primeros se resistían al cambio y, ante todo, valoraban positivamente la riqueza material, mientras que, al contrario, los segundos defendían a ultranza el cambio social y pretendían conseguir un medio ambiente seguro y limpio. Los resultados del sondeo efectuado mostraban que:

"la vanguardia ya se ha formado y se está preparando significativamente para confrontarse con la retaguardia, socialmente y políticamente, en tanto que intenta reorientar la sociedad" (Milbrath: 98),

No obstante, existían diferencias entre los distintos países analizados, dado que, por ejemplo, según sus resultados, la idea de los límites al crecimiento estaba plenamente aceptada en Alemania, bastante aceptada en Inglaterra, pero mucho más cuestionada en los Estados Unidos. 
NUEVA ESCALA DE MEDICIÓN DEL CAMBIO CULTURAL A TRAVÉS DEL NUEVO PARADIGMA ECOLÓGICO (Dunlap et al., 2000; Grendstad, 1999)

I) Sobre los limites al crecimiento:

1. Nos estamos acercando al límite en el número de personas que la tierra puede soportar.

2. La tierra dispone de muchos recursos naturales, sólo debemos aprender a explotarlos.

3. La tierra es como una nave espacial con un espacio y unos recursos limitados.

II) Sobre el anti-antropocentrismo:

4. Los humanos tienen el derecho de modificar el medio ambiente natural con el fin de satisfacer sus necesidades.

5. Las plantas y los animales tienen tanto derecho como los humanos a existir.

6. La misión de los humanos es gobernar el resto de la naturaleza.

III) Sobre el equilibrio de la naturaleza:

7. Cuando los humanos interfieren en la naturaleza, frecuentemente se producen consecuencias desastrosas.

8. El equilibrio de la naturaleza es lo suficientemente fuerte como para soportar los impactos de las sociedades industriales modernas.

9. El equilibrio de la naturaleza es muy delicado y se altera muy fácilmente.

IV) Sobre el rechazo del exencionalismo:

10. El ingenio humano asegurará que no hagamos de la tierra un lugar inhabitable.

11. A pesar de nuestras habilidades especiales, los humanos aún siguen estando sujetos a las leyes de la naturaleza.

12. Los humanos finalmente aprenderán lo suficiente sobre cómo funciona la naturaleza, de tal modo que serán capaces de controlarla.

V) Sobre la posibilidad de una crisis ecológica:

13. Los humanos están abusando de modo severo del medio ambiente.

14. Se ha exagerado mucho la llamada 'crisis ecológica' de la humanidad.

15. Si las cosas continúan como ahora, pronto experimentaremos una gran catástrofe ecológica.

Nota: en los cuestionarios el orden utilizado es $1,4,7,10,13,2,5,8,11,14,3,6,9,12,15$.

Aunque R.E. Dunlap no lo hace explícito en sus trabajos de este modo, puede interpretarse que según él, la cultura cambia porque cambia el medio ambiente y es precisamente este cambio cultural lo que pretende medir la escala HEP-NEP. No obstante, él mismo acepta que otras cosas cambian además del medio ambiente, y por lo tanto también influyen a la cultura, como son los medios de comunicación o la educación (R.E. Dunlap, 2001). 
Posteriormente, Olsen, Lodwick y el mismo Dunlap (1992) realizaron una de las revisiones más notables sobre la investigación relativa, cambio de paradigmas culturales. En aquella obra se interpretaba, bajo una perspectiva histórica hegeliana (previamente sugerida por la sociología ambiental de Allan Schnaiberg), que si bien era posible detectar dos paradigmas culturales bien distintos de tesis y antítesis, también podía ocurrir que estuviera emergiendo un nuevo paradigma de síntesis entre lo que ellos denominaban "Paradigma Social Tecnológico" (la tesis) y el "Paradigma Social Ecológico" (antítesis), una síntesis que correspondería al paradigma actual del desarrollo sostenible. Según su enfoque, un paradigma comprende tanto las creencias como los valores sobre el conjunto de la realidad que puede percibirse, siendo las creencias aquello que nos informa sobre lo que la realidad es, mientras que los valores son lo que juzga cómo la realidad debería ser (o lo bien o mal que ésta es). En opinión de Olsen et al. (1992) tales distinciones conceptuales no habían sido suficientemente abordadas en los estudios empíricos anteriores. Por ello, su trabajo pretendía operacionalizar el cambio de paradigmas realizando las siguientes distinciones: (a) entre cuatro creencias tecnológicas y cuatro creencias no tecnológicas; (b) entre cuatro creencias ecológicas y cuatro creencias no ecológicas; y (c) entre cuatro valores tecnológicos y cuatro valores ecológicos. Con ello, por un lado, pretendían determinar en qué medida el Paradigma Social Ecológico se correspondía o era particularmente distinto a una perspectiva social "post-industrial" - y que incluía, además de las creencias y valores de carácter ecológico, otras numerosas creencias y valores de tipo social, económico y político. Y por otro lado, se buscaba evaluar la consistencia entre estas creencias y valores. Sus conclusiones aseguraban que el Paradigma Social Ecológico tenía mucha menos relación con el paradigma post-industrial de lo que la literatura social había estimado hasta el momento, por lo que recomendaban mantener la distinción entre ambos. En otras palabras, no era lo mismo la "visión ecológica" del mundo, que la "visión post-industrial", aunque ciertamente se podían observar ciertas conexiones entre ambas. Al mismo tiempo señalaban que las mayores incongruencias ocurrían en aquellos sectores de población que podían estar "cambiando" de paradigma y que estarían pasando del paradigma tecnológico al ecológico. En su opinión, la resolución de las contradicciones que en buena parte mostraban ambos paradigmas hacía prever la emergencia de un nuevo paradigma de síntesis, el del Desarrollo Sostenible, y que podía caracterizarse a la vez por un conjunto de ocho "creencias sostenibles", que se oponían a otro conjunto de ocho creencias de tipo "no sostenible".

Aunque con una orientación más polítológica que sociológica, la interpretación de Ronald Inglehart sobre el post-materialismo aporta otra interesante contribución, que permite suplir, en parte, aunque no sin controversia, la falta de contenido teórico a la hora de analizar determinadas conductas y percepciones en torno al cambio y la calidad medioambientales. Según Inglehart (1990) las sociedades industriales avanzadas están atravesando un proceso de cambio 
RIS

RETISTA INTERNACIONAL DE SOCIOLOCÍA

N" 28. Enero-dbril. 2001

J. DAVIDTÁBARA

cultural profundo en el que determinados valores tradicionales, como la adhesión al crecimiento económico, el orden público o la defensa nacional están perdiendo terreno. Basándose en la teoría de Maslow, que distingue entre necesidades básicas y superiores, Inglehart argumenta que las nuevas generaciones de estas sociedades ya han podido satisfacer las primeras, por lo cada vez son más partidarias de todo un conjunto de valores (los post-materiales) que demandan mayor participación cívica, libertad de expresión y mejora de la calidad del medio ambiente. El nuevo axis cultural, pues, se encuentra entre materialismo y post-materialismo, lo cual provoca importantes presiones al sistema político vigente. A partir de este marco general, Inglehart publicó en 1995 un análisis empírico basado en sondeos de opinión, que contenía el mayor número de países consultados hasta la fecha. A partir de los datos de fuentes diversas, como el World Values Survey de 1990-91, el Eurobarómetro o la OCDE, se analizaron resultados procedentes de un total de 43 países ${ }^{11}$, que permitieron reafirmar el "cambio cultural" (cultural shift) intergeneracional que se estaba produciendo en las sociedades industriales, y especialmente entre las surgidas en la posguerra. No obstante, y reconociendo algunas de las numerosas críticas que había recibido su teoría, Inglehart acabó tomando una postura más moderada, arguyendo que lo evidente era que el cambio estaba ocurriendo, y que posiblemente formaba parte de un cambio cultural más amplio, por lo que no era necesario preocuparse excesivamente por etiquetas terminológicas u otras discusiones nominalistas (Inglehart, 1995).

Como ya se ha apuntado, la tesis de Inglehart fue rebatida por varios autores, incluyendo el mismo Dunlap. A partir de los datos recogidos en 24 países en 1992 en el Health of the Planet Survey, se llegó a la conclusión de que la riqueza nacional se correlacionaba más frecuentemente de manera inversa que directa con la preocupación ambiental mostrada por los ciudadanos de cada país, lo cual ponía en jaque la tesis postmaterialista (Dunlap y Mertig, 1994). Resultados igualmente críticos con la tesis postmaterialista basados en el mismo sondeo se hallan en Adeola (1998) en relación con los países que forman parte o no del núcleo de las sociedades industrializadas contemporáneas. Por otro lado y a pesar de que Cotgrove (1982) también coincidía con Inglehart por lo que se refiere al decisivo papel de la socialización de los jóvenes en el proceso de cambio cultural, las conclusiones del primero diferían notablemente del segundo. Cotgrove, partir de sus propios datos, ponía en cuestión el hecho de que los industriales eran individuos que también habían satisfecho sus necesidades básicas, pero que, en cambio, no mostraban demasiado interés por el medio ambiente, ocurriendo algo muy parecido con los dirigentes sindicales (no lo estaban interesados entonces, aunque sí ahora, aunque por otras razones...). De

\footnotetext{
"Algunos de estos análisis contienen datos procedentes de más de 220.000 entrevistas.
} 
este modo, la adhesión a uno u otro paradigma no podía explicarse solamente por motivos que tienen que ver con el bienestar material, sino que se explican por otras razones relativas a la propia dinámica del cambio cultural (cambio de cosmología), como son las transformaciones que padece la estructura social.

Del conjunto de las anteriores aportaciones se extrae que la investigación, mediante soporte empírico, del cambio cultural hacia nuevos paradigmas más acordes con las creencias y valores ambientales y de sostenibilidad tiene una larga tradición. Aportaciones, como la realizada por Grendstad (1999), donde se aplica, se evalúa y se cuestiona la adaptabilidad de aplicación de la escala NEP, revisada para el caso de Noruega, permiten observar que esta línea de trabajo sigue estando en plena actividad. No obstante, se trata de un campo que no ha recibido la atención que merece, quizás porque requiere tanto de un mayor conocimiento de las aportaciones realizadas por la sociología ambiental en este campo, como de un mayor dominio de las técnicas cuantitativas de investigación.

\section{¿A quien medir?}

La mayoría de los estudios de percepción ambiental tienden a tomar como universo de referencia el conjunto de la población, lo cual se corresponde con la preferencia cuantitativa que está presente en este campo de investigación. Al mismo tiempo, la elección de subpoblaciones específicas como marco de investigaciones cuantitativas ha sido objeto de crítica, debido a que no es posible aplicar muchos de los presupuestos, como el de aleatoriedad, en los que se fundamentan la extrapolación y representatividad de los resultados muestrales. No obstante, puede decirse que el supuesto contrario, y que cree posible el análisis de actitudes, comportamientos o acciones de determinados colectivos sociales a partir de muestras generales, es tanto o más criticable. Ni que decir tiene que los contrastes y las informaciones que se obtienen a partir de muestras específicamente obtenidas de grupos concretos son mucho más profundas y ricas que las recogidas a partir de muestras generales y que, a pesar de que su aparente falta de representatividad sea motivo de crítica, su uso complementario con las segundas es altamente recomendable y en algunos casos del todo necesario.

A diferencia de los estudios que toman como universo de referencia el conjunto de la población de un contexto determinado y utilizan los contrastes estructurales para explicar las diferencias entre las distintas subpoblaciones que se detectan dentro de la muestra, existe también una considerable literatura dedicada a analizar específica y solamente a grupos particulares. Aquellos trabajos que eligen a un único grupo o subpoblación específico (o complementario a las muestras generales) lo hacen de modo más frecuente en los siguientes colectivos: 
R I S

RETISTA INTERNACIONAL DE SOCIOLOGIA

N" 28. Enero-dbril. 200

J. DAYIDTIBAARA

a) Los activistas o miembros de grupos ecologistas. En este campo existe una amplia literatura, que se remonta a los años setenta y que intenta analizar, entre otras cuestiones, los motivos o la racionalidad de las acciones de estos colectivos (e.g.: Tillock y Morrison, 1979; para discusión, Tábara, 1999b) y compararlos con las del resto de la población. Grenstadt (1999), por ejemplo, combina cuestionarios al conjunto del público con uno dirigido a grupos ecologistas.

Por otro lado, Dunlap (2000), Mertig y Dunlap (1995) evalúan el éxito del movimiento ambientalista en los Estados Únidos mediante un conjunto de indicadores obtenidos con un sondeo de opinión, tales como la percepción del impacto en las políticas nacionales, las actitudes personales ante estos grupos y la aprobación de sus objetivos o el nivel de confianza que sus actuaciones inspiran.

b) Las élites politicas y los principales agentes económicos. El mayor esfuerzo internacional de carácter cuantitativo llevado a cabo en tiempos recientes sobre la percepción y el cambio cultural ambientales de las élites políticas, ha sido realizado por el proyecto GOES (2000). Algunos ejemplos de este trabajo, aunque parten o combinan encuestas al conjunto de la población son los de Worscerter (1993) y Andrews (1998), quien estudia las percepciones de líderes de grandes empresas económicas de los Estados Unidos para observar los motivos que mueven sus estrategias ambientales corporativas.

c) Las mujeres en contraste con los hombres. En este caso, existe también una sustancial labor hecha, aunque la mayoría de los estudios parten de muestras del conjunto de la población, como es el caso de Mohai (1992) y la revisión de su "paradoja" - en las que las mujeres se muestran más preocupadas pero menos dispuestas a actuar - realizada por Ozane (1999). Otros estudios elaborados en este campo son, por ejemplo en Europa, el de Carlsson-Kanyama (1999), y en África, el de Aboud (1996).

d) Los profesionales de los medios de comunicación. Este colectivo se encuentra aún muy poco estudiado, a pesar de ser clave en la construcción y percepción de los problemas ambientales y de sostenibilidad. Worscerter (1996) entrevistó a 24 periodistas británicos, encontrando que éstos tienden a subestimar el interés y las acciones que el público tiene o hace con relación a los temas ambientales. De forma similar, Tábara (1998b) planteó un cuestionario a este colectivo dentro el marco de la Unión Europea.

e) Algunas etnias particulares: los inmigrantes o no residentes. Estos sectores empiezan a ser motivo de algún trabajo empírico, facilitado por la creciente penetración de los discursos sobre el racismo ambiental y la justicia ambiental. Vaughan y Norderstam (1991) revisaron los estudios sobre las diferencias étnicas en la percepción de determinados problemas ambientales, hallando que en los EUA los colectivos de raza blanca tienden más a evaluar de manera más grave problemas como la disminución de la capa de ozono, el cambio climático o 
la lluvia ácida, mientras que los de raza no blanca se preocupaban más por los problemas tecnológicos, tales como los riesgos nucleares. Emmet (1998) retoma la misma discusión y analiza tales diferencias entre blancos y negros no encontrando ninguna distinción significativa entre la preocupación ambiental de ambos (ver también Caron, 1995; y sobre los contrastes de los hispanos ver Noe et al., 1989-90 y Bechtel et al., 1999). Los datos obtenidos en Tábara (1995) sugerían que los inmigrantes o hijos de inmigrantes tendían a valorar más negativamente el medio ambiente que el resto de la población, lo cual podría explicarse por razones claramente objetivas del lugar donde suelen residir.

f) Los niños y los jóvenes. Finalmente, este colectivo empieza a ser objeto de crecientes investigaciones. Así, por ejemplo, la encuesta efectuada por Boyes y Stanisstreet (1993), a pesar de su bajo nivel de respuesta, les permite argumentar que los niños tienden a simplificar mentalmente tanto las causas como las soluciones de los problemas ambientales (aunque también es posible que este proceso no sea exclusivo de los niños). La tesis doctoral de Grundy (1998) estudió conjuntamente, usando tanto metodologías cuantitativas como cualitativas, las percepciones ambientales de padres e hijos en Escocia, descubriendo que los hijos no eran más "ecologistas" (ambientalmente activistas) que los padres ${ }^{12}$. De carácter cualitativo, es el estudio de los jóvenes entre 15 y 17 años, de Hillcoat et al. (1995).

De este panorama general, se puede ver, pues que el abanico de posibilidades para el estudio de las percepciones ambientales de colectivos particu-lares es inmenso y que, en todo caso, es complementario al estudio de grandes poblaciones en su conjunto.

\section{¿Cómo medir?}

Como vemos, la lista de decisiones estratégicas a las que un investigador social se enfrenta en el momento de llevar a cabo sus trabajos de campo en este área empieza ya a ser bastante extensa, a lo cual se suman ahora las inevitables cuestiones relativas a escoger entre un diseño cuantitativo u otro de carácter cualitativo.

\section{Metodologías cuantitativas}

La mayoría de los trabajos empíricos en sociología que pretenden medir la percepción ambiental de grandes poblaciones ha tendido a la utilización de sondeos cuantitativos, vía sondeos aleatorios estratificados ejecutados

\footnotetext{
12 Lo cual pone en entredicho si tiene algún sentido esperar demasiado que las generaciones futuras hagan por el medio ambiente lo que nosotros no estamos dispuestos a hacer ahora.
} 
RIS

RETISTA INTERNACIONAL DE SOCIOLOCIA

Na 28. Enero-Abril. 2001

mediante entrevistas personales o telefónicas. De hecho, casi todos los trabajos mencionados en esta revisión tienen este carácter. Tanto si el objeto ha sido medir el grado de "preocupación" relativa, el nivel de conocimiento u otras opiniones diversas en relación con determinados problemas ambientales, como si se han buscado indicios de cambios culturales más profundos, el método cuantitativo ha tendido a ser el predominante ${ }^{13}$.

En algunos estudios, como el mencionado de Inglehart (1995), se agregan datos de varios sondeos distintos, lo cual puede ser objeto de ciertas críticas. En otros, como el realizado por el Instituto Internacional George H. Gallup en 1992 bajo la cordinación de Riley Dunlap, fue posible la realización de un único diseño aplicable a un gran número de poblaciones, constituyendo el mayor trabajo sobre la percepción de los problemas ambientales realizado hasta la fecha: The Health of the Planet Survey, también conocido como el Global Survey. En aquella ocasión, se recogieron datos a partir de un total de veinticuatro países tanto de la órbita occidental, como de Asia, África o América Latina ${ }^{14}$. A partir de sus resultados, Dunlap et al. (1993) afirmaron que, en tiempos de la Conferencia de Estocolmo de 1972, los problemas ambientales eran entendidos principalmente como temas estéticos o amenazas a la belleza de la naturaleza, pero veinte años más tarde existía una creciente conciencia de que tales problemas constituyen ya amenazas a la salud humana. Según este autor, este sondeo demostró la existencia de un consenso prácticamente general sobre la realidad de los problemas ambientales y la baja calidad ambiental del planeta. Basándose en las mismas preguntas realizadas por Dunlap, la organización canadiense Environics Internacional realizó otro sondeo público internacional, demostrando en 1997 que la preocupación mundial desde entonces se había incrementado (Collins, 1997).

\footnotetext{
${ }^{13}$ No obstante, esta preferencia cuantitativa no ha hecho irrelevante el uso de técnicas cualitativas, dado que la mayoría de cuestionarios y análisis previos a la ejecución de estos trabajos cuantitativos se fundamentan en la realización de grupos de discusión u otras técnicas de carácter cualitativo (Weber y Corrado, 1993).

${ }^{14}$ A pesar de los matices y las diferencias por países, fue posible extraer varias regularidades que aparecían en casi todas las naciones. Así, la calidad del agua y del aire constituyeron dos de los problemas de mayor preocupación; la mayor parte de la población entrevistada creía necesario mejorar la cooperación internacional con el fin de proteger el medio ambiente de los paises menos desarrollados; la mayoria de los países afirmaron que los cambios ambientales afectarán la salud de sus hijos y sus nietos en los próximos veinticinco años; y la preocupación por el medio ambiente no aparecía en absoluto como un lujo exclusivo de los países más desarrollados o industrializados (Dunlap, 1992, 1993 y 1994). Para una comparación de los datos obtenidos en cinco países a partir del International Social Survey Program (ISSP), ver J.D. Skrentny (1993).
} 
En estos trabajos cuantitativos, la agregación de opiniones se efectúa por lo común mediante preguntas cerradas cuyas respuestas están tabuladas en escalas Likert de cuatro o cinco valores sobre el nivel de acuerdo o desacuerdo (por ejemplo, nada, poco, bastante, muy) o más detalladamente en valores numéricos que van del 0 al 9. En algún trabajo, como el mencionado de Dunlap (1992), el cuestionario empezaba con una pregunta abierta al principio, relativa a saber qué problemas en general preocupaban más al entrevistado, sin haberle informado anteriormente que le iban a hacer una encuesta sobre temas de medio ambiente. Ello se hacía para evitar un fenómeno que se ha detectado en este campo, y que podríamos denominar como "perjuicio ambiental positivo" - todo el mundo se vuelve muy ecologista cuando responde preguntas solamente de tipo ambiental y no se conectan con otras problemáticas sociales y económicas. Por otro lado, el uso de escalas más complejas, como la facilitada por Dunlap y sus colaboradores con relación a los paradigmas PEH-NPA, se hace imprescindible para poder conectar la teoría con la labor empírica en este terreno. Por el momento, la creación de escalas nuevas es poco recomendable, dado que impide la comparación de resultados, y supone un reto metodológico y teórico que dificilmente puede superar las propuestas facilitadas por los grandes maestros en este campo.

A partir de los años ochenta, un buen número de observadores, principalmente psicólogos, planificadores y geógrafos, empezó a profundizar en las limitaciones de los trabajos de carácter más cuantitativo, los cuales topaban no sólo con importantes escollos de tipo metodológico, sino que además aportaban resultados que parecían excesivamente pobres o desconectados de la teoría y cuya relevancia era más que dudosa. En algunos círculos de las ciencias sociales ambientales se ha producido una lógica y creciente crítica a estos enfoques cuantitativos por su superficialidad y por el hecho de que si bien pueden dar lugar a resultados representativos, no pueden aportar conclusiones sobre lo que piensa la gente, sino sobre lo que no ha tenido tiempo de pensar. Esto es especialmente cierto en materia de Cambio Ambiental Global (Tábara, 2000a). La necesidad de encontrar metodologías estables para obtener datos comparables en el tiempo, lo cual era prometido por las técnicas cuantitativas, también ha provocado sus riesgos. En los años setenta y ochenta (y en España en los noventa), justo cuando se creía haber encontrado un "filón" para la actividad investigadora y se acordaban unos procedimientos consensuados para obtener resultados suficientemente válidos y representativos, parecía como si se hubiera vuelto irrelevante profundizar en la innovación de técnicas para mejorar los resultados y explicaciones de este tipo de investigación. Desgraciadamente, sería la misma falta de imaginación metodológica lo que ponía en peligro un campo que acababa sólo de empezar. El compromiso excesivo con unas metodologías muy específicas o "normales" (en el sentido de Thomas Kuhn) habría impedido la transdisciplinariedad y la integración y, con ello, se habrían reducido las posibilidades para un pleno desarrollo de este campo de investigación. 
RIS

RETISTA INTERNACIONAL DE SOCIOLOGIA

N" 28. Enero-duril. 2001

J. DAVID TABARA

\section{Metodologías cualitativas}

Si bien la antropología y la psicología ya habían efectuado un encomiable trabajo cualitativo en materia de percepción ambiental en los años sesenta y setenta (Whyte, 1977), la sociología ha tendido a descuidar bastante la utilización de metodologías cualitativas en este terreno, si bien este tipo de técnicas empieza a tener una creciente utilización en los últimos años. El motivo de este "redescubrimiento cualitativo" se debe a varias razones, entre las cuales cabe destacar el mayor reconocimiento de las limitaciones que son endémicas a los procedimientos cuantitativos, la mayor comprensión de la naturaleza de los problemas de medio ambiente, y el creciente rechazo de los presupuestos individualistas que creen en la idoneidad de la agregación de opiniones atomizadas como base para comprender opiniones sobre cuestiones colectivas complejas, como son las de carácter ambiental. Tratándose de una materia relativamente apartada de los problemas cotidianos, la formulación de opiniones mínimamente fundamentadas por parte de la población sobre los presentes problemas ambientales requiere de un mínimo de información, de diálogo y de tiempo de reflexión, que difícilmente se dispone en los procedimientos cuantitativos. Además, la creencia de que es suficiente o adecuado agregar y extrapolar opiniones individuales generadas en un momento dado de manera aditiva, sin tener en cuenta los procesos de formación colectiva de opinión, parece cada vez más dificil de sostener. Una referencia ineludible en este campo son las aportaciones de Burgess et al. (1988a y 1988b) en lo que refiere a la percepción local del medio ambiente y de Burgess et al. (1995) en su estudio cualitativo y comparativo del Reino Unido y Holanda en relación a la percepción social del cambio climático.

En este sentido la utilización de grupos de discusión formados por individuos seleccionados a partir de sistemas de reclutamiento basados en cuotas de heterogeneidad, está recibiendo recientemente una notable atención, dado que permite la creación de espacios de reflexión colectiva mucho más acordes con las dimensiones temporales, espaciales y morales que plantean los problemas ambientales y de sostenibilidad. Estas aproximaciones parecen especialmente útiles para plantear cuestiones complejas, como las que aborda la Evaluación Ambiental Integrada y que se comentan más adelante (Dürrenberger et al., 1997; Macnaghten et al., 1995; Jaeger et al., 1999 y Tábara y Querol, 1999).

\section{De la triangularización a la integración}

Si bien el futuro de los estudios sobre la percepción ambiental basados solamente en análisis cuantitativos es muy incierto - por no decir difunto, en tanto no se complemente con análisis cualitativos u otras técnicas de investigación (triangularización) y se conecte lo empírico con la teoría macrosocial-, los 
desarrollos metodológicos más interesantes se producen en aquellas "periferias" (por usar el vocabulario de Lowenthal), o en aquellas intersecciones, que están siendo capaces de abrir ciertas barreras disciplinares y que llevan a cabo la interpretación teórica de las concepciones de los problemas del medio ambiente de manera integrada, incluso con las aportaciones de las ciencias naturales. Aunque el concepto general de integración es multidimensional, la integración metodológica tiene un significado más restringido, que se refiere a la posibilidad de encontrar modos de incorporación de conocimiento entre las diferentes fuentes de producción del saber, sean éstas expertas o no, procedan de las ciencias sociales o de las ciencias naturales. El nuevo campo de la Evaluación Ambiental Integrada (EAI) (Tábara, 1999, 2000 y 2001b; Tábara y Querol, 1999) avanza en esta dirección, si bien su propósito explícito principal no es tanto obtener la puridad o la distinción académicas, como conseguir resultados relevantes. En particular, la EAI Participativa considera decisiva la incorporación de las perspectivas de los agentes o las percepciones de los actores con intereses en juego (stakeholders). La búsqueda de un conocimiento ambiental que no sólo sea eficaz para la mejora de la calidad del medio ambiente y la sostenibilidad, sino que también sea equitativo en términos sociales, constituye la razón de ser de este nuevo enfoque metodológico. En este sentido, la integración de conocimientos y opiniones por parte de individuos que tienen capacidades dialógicas y que usan medios y lenguajes de expresión muy distintos, hace cada vez más necesario el uso de diseños cualitativos innovadores. Así, el uso y la interpretación de collages sobre visiones y escenarios de futuro (Kasemir et al., 2000) o de la construcción participativa de tipos ideales sobre variables, funciones o desarrollos sociales sobre medio ambiente y sostenibilidad (Tábara $e t$ al., 2000), se erigen como aproximaciones originales en este terreno.

\section{¿Para qué medir?}

Finalmente, llegamos a la espinosa cuestión de cómo justificar los recursos y esfuerzos que supone llevar a cabo un trabajo empírico en esta materia. Si bien es cierto que la ejecución de muchas investigaciones sobre opinión pública responde a motivos de carácter institucional o profesional que escapan al presente análisis, podemos encontrar, al menos, dos grandes clases de razones que, a mi entender, legítimamente justifican la necesidad o la conveniencia de llevar a cabo estas tareas. Las primeras son de carácter estrictamente académico, orientadas a la mejora del conocimiento científico, mientras que las segundas, de naturaleza política, pretenden la democratización o la mejora de la eficiencia de las actuaciones públicas y de los procesos de decisión en materia ambiental. En este segundo caso, se trata de establecer un canal de comunicación entre la ciudadanía, una parte lo más representativa de ella, y los responsables de la política ambiental y de las acciones orientadas a la mejora de la sostenibilidad. 
R IS

RETISTA INTERNACIONAL DE SOCIOLOGIA

№ 28. Enero- + bril. 2001

Evidentemente, ambas funciones - la académica y la política - no son incompatibles y un buen diseño metodológico puede, o debería poder, producir resultados que sirvan para ambos objetivos.

\section{Relevancia académica}

En el ámbito académico, la utilización de un diseño empírico se justifica en tanto que permite mejorar y especificar con mayor detalle la interpretación de las causas sociales que explican la percepción, o la falta de percepción, de los problemas ambientales o la existencia, o inexistencia, de determinadas creencias, valores o actitudes culturales que mediatizan la concepción del entorno en un contexto dado. Por tanto, a mi entender, estos trabajos deberían informar y dar cuenta de rasgos relativos al cómo las poblaciones respectivas conciben los distintos problemas ambientales y a qué causas culturales responden, y dar cuenta de las características sociales y estructurales más relevantes que permiten observar los principales contrastes entre las distintas subpoblaciones que las componen. El objeto aquí no es otro que el de mejorar el conocimiento como valor propio, independientemente de, o dejando en un segundo plano, los usos a los que se destinará este conocimiento.

No obstante, si como ocurre en este caso, el objetivo de escoger una aproximación empírica para el estudio de las percepciones ambientales no es político, sino "puramente" científico, no tiene sentido que estos estudios se realicen de un modo completamente desconectado de la teoría. A mi parecer, el trabajo empírico no puede desligarse de la teoría, sino que ha de contribuir a ella, mejorándola, especificándola, y en su caso, rechazándola. No basta con describir. El continuo análisis, comparación, síntesis y verificación teórica se hacen de todo imprescindible en el ámbito académico. Ya no se trata, $-\mathrm{y}$ en especial en los diseños cuantitativos-, de que los resultados obtenidos por tales trabajos sean comparables a lo largo del tiempo y entre poblaciones procedentes de contextos distintos, sino que también sean contrastables con otros resultados y corroborables por las teorías existentes. Consiste, pues, en obtener datos acumulables y que se correspondan con enfoques teóricos más o menos aceptados dentro de la disciplina (cualquiera que sea la orientación política) y que integren los desarrollos alcanzados hasta el momento en esta materia, y de este modo evitar esfuerzos redundantes. Por ello, y si aceptamos esta posición más rigurosa, podemos observar cómo muchos trabajos que actualmente se hacen pasar por obras de gran talla académica en este campo, en realidad constituyen obras mucho más modestas, con prácticamente ningún contenido teórico.

\section{Relevancia politica}

El estudio y la puesta a la luz pública de los resultados de investigaciones 
sobre opinión publica, siempre y cuando se garantice su máximo rigor e independencia, pueden ser una forma muy válida de democratizar los discursos públicos en general. Lo mismo se puede argüir en lo que concierne a las decisiones relativas a la mejora de la calidad del medio ambiente y de la sostenibilidad. Así, si concebimos que una buena parte de la investigación social tiene un impacto que va mucho más allá de sus límites académicos, entonces podemos convenir que las ciencias sociales pueden llevar a cabo unas funciones democratizadoras y reflexivas notables, que no pueden realizarse mediante otras vías. Si al fin y al cabo, se reconoce que el conocimiento aportado por los estudios de opinión va a ser utilizado de todos modos, quizá es hora ya de empezar a pensar en algunos criterios para que tal conocimiento sea además, y por acuerdo democrático, también útil. Sin embargo, cuando el propósito explícito es informar al proceso político, la utilización única de metodologías cuantitativas que no desvelan las preferencias informadas o, reflexiones de los ciudadanos ante una problemática tan compleja y se limita al análisis de "impresiones", es altamente peligrosa, tanto para la sociedad, como para el futuro del medio ambiente.

De esta manera, la realización de sondeos ha llegado a erigirse no sólo en un medio probadamente útil para el conocimiento de las opiniones de los ciudadanos sobre un sinfin de cuestiones sociales, sino también en una arma eficaz para defender las reivindicaciones de multitud de entidades públicas o privadas ${ }^{15}$. Raro es el caso de resultados procedentes de estudios que se presentan como representativos, que no tengan una lectura política. Y los políticos y la sociedad civil lo saben. Por ello, a menudo, ambos llevan a cargo o financian sus propios estudios sobre los mismos temas y sobre las mismas poblaciones, por lo que no es de extrañar la aparición de resultados divergentes o contradictorios. Un ejemplo de esta función de los sondeos, bien como mecanismo eficaz de crítica social o como plataforma de legitimación en temas de medio ambiente, puede contemplarse en los resultados publicados en el periódico británico The

\footnotetext{
${ }^{15}$ Como es sabido, la credibilidad de los sondeos de opinión mejoraron sustancialmente a partir de los aciertos obtenidos por el investigador estadounidense George H. Gallup en las elecciones presidenciales de 1936, 1940 y 1944. A pesar de algunos errores posteriores y de las críticas recibidas por parte de otras corrientes dentro de la sociología, lo cierto es que los sondeos de opinión se han erigido en un instrumento casi imprescindible, tanto en los análisis y campañas políticas, como en los estudios de mercado. No obstante, la finalidad que Gallup asignaba a los sondeos no se limitaba a estas aplicaciones. Según él, muchas de las propuestas o críticas que surgían de la investigación social deberian ser puestas a escrutinio público mediante un método fiable que recogiera las visiones de los ciudadanos sobre ellas, y de esta forma, que permitiera aumentar la democratización de las decisiones politicas. En la actualidad, su prestigioso instituto constituye una organización internacional sin ánimo de lucro que trata temas muy diversos como la educación, la salud, los valores sociales, la religión y el medio ambiente.
} 
R IS

RETISTA INTERNACIONAL DE SOCIOLOGI.

N" 28. Enero-Abril. 200

Independent de 11 de Abril de 1997, en plena campaña electoral. Una coalición de 44 grupos de presión, cuyo número de afiliados superaba los dos millones cuatrocientos mil miembros, financió una investigación llevada a cabo por el Instituto MORI de Londres, con el fin de dar a conocer el estado de opinión sobre temas que suelen recibir poca atención por parte de los principales líderes políticos, tanto laboristas como conservadores. Se trataba de la Real World Coalition, fundada por el famoso líder ecologista y anterior director de los Amigos de la Tierra Jonathon Porrit, y que contaba con el apoyo de organizaciones como Oxfam, Christian Aid o Save the Children Fund. Entre los datos ofrecidos destacaba el hecho de que tres cuartas partes del público británico señalaba como importante aplicar políticas ambientales mucho más duras, reducir el tráfico por carretera, disminuir el consumo de energía para abatir el cambio climático, y aplicar mayores medidas de conservación a los espacios libres de especial interés natural o científico. Sin embargo, el estudio no complació a todas las organizaciones no gubernamentales que lo financiaron, lo cual daba ciertas garantías de la independencia del encargo, aunque sí permitió dar cuenta de opiniones que difícilmente llegan a la discusión política de otro modo. Es mediante estrategias como ésta, que los sondeos revelan realidades sociales, que son, al fin y al cabo, las únicas realidades que cuentan a nivel político ("opino, luego existo"; Tábara, 1997). No obstante, y de todos modos, en temas ambientales muy complejos los estudios de opinión pública suelen ser ignorados por los responsables políticos en cuestión ${ }^{16}$.

\section{De los resultados utilizados a los resultados útiles}

Al mismo tiempo, la investigación sobre la percepción ambiental puede enmarcarse dentro de una discusión mucho más amplia y que está presente dentro de los debates de la sociología y las ciencias ambientales contemporáneas. En particular consiste en reflexionar si tiene sentido distinguir entre conocimiento sobre el medio ambiente (y en nuestro caso, sobre su realidad social) y conocimiento para el medio ambiente (y en nuestra área, para la mejora de la capacitación ambiental de la sociedad), discusión que a la vez puede conectarse

\footnotetext{
${ }^{16}$ Por ejemplo, en el caso del cambio climático, Löfstedt (1995) señala cuatro razones por las cuales los estudios de opinión son ignorados por los decisores politicos, los cuales tienden a creer que: (a) las encuestas de opinión no son suficientemente precisas; (b) lo que más interesa al público es lo económico; (c) los estudios de opinión son innecesarios en materia ambiental, dado que los problemas ambientales pueden resolverse de manera técnica y experta; (d) los resultados de estos estudios sociológicos pueden dar mala reputación a todos los esfuerzos politicos por conservar energia.
} 
con la distinción anterior entre conocimiento útil y conocimiento utilizado. Los estudios sobre percepción ambiental pueden justificarse, y de hecho así ocurre a menudo, sólo porque aportan un conocimiento sobre la realidad socio-ambiental, aunque su finalidad última no sea su utilización explícita para la búsqueda de soluciones a los problemas ambientales. Tales estudios pueden ser útiles o utilizados en función de determinados propósitos tanto científicos como políticos, aunque en todo caso, y como hemos visto, será necesario disponer de algún criterio para evaluar ambas funciones. Sin embargo, no podemos presuponer que un estudio sobre la percepción ambiental acabe siendo necesariamente ítil para el medio ambiente, aunque sea utilizado por los responsables de gestionarlo para conocer la apreciación por parte del público de sus éxitos o fracasos o para modificar tales percepciones en lugar de solucionar los problemas que deberían abordar (e.g. "mejorar" las percepciones, en lugar de "mejorar" el medio ambiente). La transformación de un resultado simplemente utilizado en un resultado verdaderamente útil para el medio ambiente y la sostenibilidad probablemente supondría un cambio de los planteamientos que guían la investigación actual y sustituirlos por otros muy distintos, aunque no menos rigurosos (y que probablemente no dependerían sólo de las decisiones del investigador). Consistiría en avanzar en una dirección de la ciencia y de la producción e integración del conocimiento, que permitiese descubrir, eso sí, lo más objetivamente posible, las limitaciones y oportunidades en las que el universo social se encuentra para abordar los problemas ambientales con los que nos enfrentamos y sus consecuencias. Evidentemente, tal propósito no trataría, bajo ninguna instancia, de politizar la ciencia o limitar la libertad de innovación, sino de encontrar un equilibrio entre la relevancia académica y el servicio a la mejora ambiental. En particular, y en lo que se refiere a los estudios de opinión publica ambiental, éstos pueden tener, y quizás debieran tener, una función reflexiva muy importante en la dirección de constatar las graves dificultades culturales y educativas con las que nos enfrentamos para adaptarnos y prever adecuadamente la aceleración exponencial del cambio ambiental negativo y poder actuar así en consecuencia (Tábara, 2001a).

\section{ALGUNAS INVESTIGACIONES EN ESPAÑA}

La sociología española cuenta ya con un buen número de trabajos que permiten observar algunas tendencias y patrones destacables en lo que se refiere a los objetos, resultados y metodologías de medición de la percepción social del medio ambiente. Si bien en este artículo pretende llevar a cabo una revisión exhaustiva de todo lo, que se ha realizado y se está realizando en estos momentos, sí pretendo en las siguientes líneas poner de relieve algunos de los principales datos, potencialidades y debilidades de los estudios efectuados por 
RIS

REIISTA INTERNACIONAL DE SOCIOLOGI.A

N" 28. Enero-Abril. 2001

J. DAYID TÁBARA

equipos y autores diversos en España. El propósito no es otro que contribuir, en la medida de lo posible, a la consolidación de este campo de investigación cuyo interés es, sin duda alguna, creciente.

La investigación empírica de las percepciones ambientales en España se ha adaptado al marco y a las posibilidades institucionales y económicas existentes en el país. Así, por un lado, se han realizado ya varias investigaciones de tipo cuantitativo en distintas Comunidades Autónomas, como es el caso de Andalucía (IESA-Andalucía, 1996; Navarro, 2000), Cataluña (Marketing Systems, 1996; Tábara, 1995 y 1996), Menorca (Tábara, 1998) o Valencia (Almenar et al., 1998). Por el otro, se ha elaborado un número relativamente reducido de estudios que tienen como universo de referencia el conjunto de la población española. A mediados de los ochenta, el Instituto IDES (1986), por encargo de la entonces Dirección General de Medio Ambiente, realizó uno de los primeros sondeos específicamente orientados a explorar la opinión pública ambiental a nivel estatal, sondeo que suele ser ignorado en los estudios que se realizan actualmente sobre esta materia.

Las fuentes de datos para los trabajos de ámbito estatal han procedido, sobre todo, del Centro de Investigaciones Sociológicas (CIS) y del Centro de Investigaciones de la Realidad Social (CIRES). Así, por ejemplo, el trabajo de García Ferrando (1991) utilizó datos procedentes de cuatro encuestas del CIS realizadas en los años ochenta; Chulià (1995) intentó discernir la conciencia medioambiental de los españoles a partir de los resultados obtenidos por el CIRES en 1994, concepto que Navarro Yáñez (1998 y 2000) retomó para realizar, sobre datos de una encuesta del IESA (1996), una tipología del apoyo ambientalista en Andalucía y demostrar cómo tales comportamientos están correlacionados positivamente con el sentimiento de competencia y cultura políticas de los ciudadanos. Bajo un marco teórico similar, el notable trabajo de Gómez Benito et al. (1999) se basa en una encuesta del CIS de 1996 y destaca por la inclusión de un modelo que relaciona actitudes y comportamientos ambientales, considerando la consistencia entre unos y otros; en dicho estudio se consideran las actitudes basándose en un conjunto de seis variables sociodemográficas y otras cuatro dimensiones ambientales relativas a la preocupación, el conocimiento, las disposiciones y unos valores básicos, distinguiéndose entre acciones colectivas e individuales. El trabajo de Martín (1999) destaca también por ser uno de los pocos casos de comparación temporal, fijando hasta seis momentos distintos, desde 1992 a 1996, a partir de los datos del CIS, del CIRES y el Eurostad. El estudio de Almenar et al. (1998) sobre la percepción ambiental en el caso valenciano sobresale, entre otras razones, por contener datos tanto de tipo cuantitativo como cualitativo.

El conjunto de resultados que es posible extraer de todos estos estudios es considerable y en la mayoría de los casos se ha abordado conjuntamente tanto las estimaciones sobre la gravedad de los problemas ambientales y la preocupación 
pública que suscitaban, como las posibles actividades y disposiciones a actuar al respecto. Así, por ejemplo, ya en el trabajo del IDES de 1986, el 89,6\% de los españoles respondía que "el incremento de la contaminación y la degradación del medio ambiente pueden ser perjudiciales para la salud e incluso para la supervivencia de la humanidad". El estudio del CIS de 1996 permitía observar que más de dos terceras partes de la población entrevistada creía que el desarrollo económico es compatible con la conservación de la naturaleza, creencia que era mantenida especialmente en las poblaciones urbanas. Los datos obtenidos por ese mismo centro en marzo del 1999, estimaban que el $64 \%$ opinaba que la conservación del medio ambiente es un problema inmediato y urgente (porcentaje menor al 72,3\% de 1996), siendo la escasez de agua el más inmediato y urgente (de una lista de cinco problemas propuestos) y los incendios el problema que recibía un mayor porcentaje de valoración "muy o bastante importante" (el 98\%) (CIS, 1996 y 1999).

Los temas relativos a la ciencia y sus relaciones con el medio ambiente, al igual que la cuestión de la energía nuclear, también han sido objeto de investigación por parte del CIS (1997a y 1997b). En el primer caso, la población española muestra un cierto pesimismo y desconfianza sobre los impactos de la biotecnología en el medio ambiente; el 53\% de los entrevistados opinaban que estos avances son muy o bastante peligrosos. Por otro lado, se percibía que las medidas de seguridad de la energía nuclear son insuficientes (58\%) y que los riesgos de almacenar o transportar los residuos radioactivos son altos o muy altos (65\%). Asímismo, es de destacar que el estudio del CIRES de 1994 corrigió algunas importantes deficiencias presentes en su diseño de 1992, e incorporaba un interesante procedimiento para averiguar el conocimiento público de la problemática ambiental (mediante el reconocimiento de la veracidad o falsedad de determinadas afirmaciones), produciendo resultados que, aunque eran de esperar, son más que desesperanzadores (Chuliá, 1995).

En la actualidad, están proliferando estudios sobre percepción ambiental llevados a cabo por instituciones y empresas varias, como es el caso de entidades como la Fundación Entorno (2000), la Empresa Nacional de Residuos Radioactivos o la Organización de Consumidores (CIS, 1996, 1997 y 1999; CIRES 1992 y 1994, ICPS, 1995 y ss.; ENRESA, 1998). Ni qué decir tiene que los motivos subyacentes que llevan a estas organizaciones tan diversas a conducir sondeos en este campo son muy distintos, aunque en más de una ocasión puedan producir resultados coincidentes o a veces sorprendentemente inesperados para las instituciones que los financian. Así, por ejemplo, en el estudio de la Empresa Nacional de Residuos Radioactivos se da el dato de que según la opinión pública española, entre seis tipos de energía posibles, la nuclear se entiende como una de las menos útiles, sólo un poco por encima del carbón y por debajo la energía solar, y eso a pesar de que el $89,6 \%$ de los entrevistados en ese mismo estudio opinaba que las centrales nucleares son extremadamente o muy 
RIS

RETISTA INTERNACIONAL DE SOCIOLOGI.

N" 28. Enero-Abril. 2001

J. DAVIDTABARA

peligrosas (ENRESA, 1998).

De los estudios consultados podemos entrever ya las siguientes características de la investigación empírica en materia de percepción ambiental en España:

1. Fragmentación y dificil comparabilidad, tanto con los trabajos realizados a nivel internacional como entre las diferentes Comunidades Autónomas u otros ámbitos territoriales del Estado.

2. Falta de sintesis y debilidad teórica, ya que prácticamente no se han conectado hasta la fecha los trabajos empíricos con las escalas o teorías sociológicas existentes en estos momentos sobre cambio cultural o cambio socioestructural derivado del cambio ambienta ${ }^{17}$.

3. Preferencia cuantitativa, dejando en muchos casos se dejan al margen los procesos de triangulación que deberían ser fundamentales al tratar una materia tan compleja y a la vez frecuentemente percibida como "distante", como son los problemas ambientales.

4. Irregularidad, ya que la falta de series temporales regulares hace prácticamente imposible realizar comparaciones a lo largo del tiempo, salvo en algún caso muy excepcional, por períodos cortos y haciendo uso de bases de datos muy limitados (Martín, 1999).

5. Falta de integración, tanto entre disciplinas científicas, como entre las esferas de acción política y cívica (Tábara, 1999 y 2000), lo cual da a lugar que los resultados obtenidos tengan escasa relevancia.

Así pues, en general en España la sociología ambiental se ha centrado en estudiar la percepción de determinados problemas ambientales y sus interacciones sociales, dejando al margen el estudio de paradigmas culturales, y lo ha hecho ante todo de manera cuantitativa. Hasta la fecha se han relegado a un segundo plano los intentos de aplicar un análisis más profundo de síntesis sobre la base de escalas como las proporcionadas por Dunlap, Catton y Van Liere o a partir

\footnotetext{
${ }^{17}$ No obstante, existe ya algún intento en esta línea, concentrado en explicar las bases sociales de la preocupación o relacionar las actitudes ambientales con los comportamientos (Navarro Yáñez, 1998; Gómez Benito et al., 1999) o en llevar a cabo análisis a partir de teorías derivadas de la psicología, la psicología social o las teorías de evaluación y gestión de riesgos (Díaz, et al., 1999). Por otro lado, y a pesar que Gómez et al., opinan que el Nuevo Paradigma Ecológico de Dunlap y sus colaboradores "constituye, hasta el presente momento, la principal aportación de carácter explicativo a la teoría del ambientalismo $[\mathrm{y} . .$.$] una teoría que ha contribuido a guiar la investigación sobre los valores$ y el comportamiento ambiental de la población en los paises occidentales" (1999: 12-13) éstos autores no utilizan la escala NPE (o NEP en inglés) en ningún momento en sus análisis de los datos recogidos por el CIS en 1996 ni tampoco el marco teórico revisado por Olsen et al., (1992) donde se distingue entre creencias, valores y actitudes. De Castro (2001), de modo muy notorio, comenta la aplicación de la escala HEP-NEP por parte de González y Amérigo en un estudio de 1998 a una muestra de estudiantes de Madrid, Segovia y Toledo, así como su uso actual para el estudio del voluntariado ambiental por parte de De Castro.
} 
de teorías macrosociológicas con contenido ambiental, como es el caso de la modernización ecológica, la teoría de la sociedad del riesgo de Urlich Beck o el postmaterialismo de Ronald Inglehart ${ }^{18}$. Esto, sin embargo, sí ha ocurrido en otros países, como nos muestra el reciente trabajo de Grendstad (1999) aplicando y adaptando la escala HEP-NEP al caso de Dinamarca. Por ello, se hace necesario profundizar mucho y con una perspectiva constructiva, pero a la vez crítica, en las aportaciones realizadas hasta el momento, puesto que si, como ya decía Boudon, la metodología es la crítica a la teoría, no podemos olvidarnos de conectar continuamente y críticamente el trabajo empírico con los análisis teóricos. De hecho, el ambientalismo desde siempre ha sido, y sigue siendo, crítico con la sociedad y el contexto social que le rodea. Sería contradictorio generar unas ciencias sociales ambientales que no sean críticas con los resultados y los procesos que ellas mismas obtienen y usan, puesto que solamente a partir de esta crítica se podrán superar muchas de las dificultades que impide la consolidación en España de estas nuevas líneas de investigación.

\section{CONCLUSIONES}

El creciente interés por parte de una diversidad de instituciones académicas, económicas, políticas o cívicas por llevar a cabo estudios empíricos dirigidos a medir la percepción social de la problemática ambiental hace necesario realizar un balance de las potencialidades, limitaciones y resultados obtenidos hasta la fecha en este campo de investigación. Con esta motivación de fondo, el propósito del presente artículo ha sido doble. Por un lado, se ha pretendido trazar un mapa general de las cuestiones metodológicas, los datos y autores más relevantes que han confluido, y confluyen, en la investigación de la percepción ambiental desde la sociología; y por otro, se ha intentado poner de manifiesto las principales dificultades y debilidades con las que se encuentra actualmente el desarrollo de esta línea de trabajo, con particular interés en el caso español. En ningún caso se ha pretendido realizar un vaciado exhaustivo y comparativo de todas y cada una de estas aportaciones, lo cual representaría un programa de trabajo que está lejos de los propósitos y los medios de esta contribución. No obstante, la revisión y el análisis efectuado aquí permiten obtener un conjunto de conclusiones que pueden ser relevantes tanto para aquellos investigadores que se adentren por primera vez en este terreno, como para aquéllos que conozcan ya algunos de

\footnotetext{
${ }^{18}$ Aunque en este último caso, España sí entraba en la lista de los 43 paises que Inglehart utilizaba para su trabajo de 1995 (Inglehart, 1995).
} 
los principales debates que actualmente se tercian en las ciencias sociales ambientales contemporáneas.

En la investigación de la percepción ambiental convergen diversas disciplinas con presupuestos y metodologías distintos, lo cual también ha dado lugar a resultados muy dispares, algunos irreconciliables y sin posibilidad de integración. No obstante, a mi entender, los trabajos en este campo sufren de unas debilidades que no son únicamente específicas de este área de trabajo, sino que afectan al conjunto de las ciencias sociales ambientales. Se trata de una serie de dilemas endémicos, y sin resolver, que surgen ineludiblemente desde el primer momento que se plantea abordar al medio ambiente desde una perspectiva social. Estas tensiones, que condicionan tanto los diseños metodológicos, como la producción y el uso de los resultados obtenidos, son las siguientes:

(a) La discusión entre realistas ecológicos y construccionistas sociales, relativa a la ontología o la existencia última de los problemas ambientales (si éstos son reales o socialmente construidos). La mayoría de los métodos y analistas sociales que abordan el medio ambiente deben "confesar" sus presupuestos de partida al respecto a la hora de realizar sus trabajos si no quieren caer en feroces críticas por parte de uno u otro extremo. Este dilema está siendo superado, en parte, en los momentos presentes, dado que tanto unos como otros evitan caer en extremismos (Buttel y Taylor, 1992; Dunlap y Catton, 1994a; Hannigan, 1995)

(b) El debate sobre si el cambio ambiental se entiende como causa, efecto o es independiente del cambio y reestructuración social este debate está representado por la discusión entre determinismo ecológico versus autonomía cultural y social (Alexander y Smith, 1996; Beck, 1992; Douglas y Wildasky, 1982).

(c) La tensión epistemológica acerca de cuál debería ser el mejor procedimiento para obtener el mejor conocimiento para enmarcar y abordar problemas ambientales complejos como el cambio ambiental global. En esta arena se enfrentan los metodológicamente populistas, que defienden el uso de metodologías participativas u otros procedimientos de integración y de creación plural del conocimiento científico, los metodológicamente elitistas, que califican de irrelevante e innecesario el conocimiento no experto en estas materias (Funtowicz y Ravetz, 1991; Janasoff y Wynne, 1992 y 1994).

(d) La discusión sobre si las ciencias sociales ambientales pueden o deben desarrollarse de manera independiente o solamente en conjunción con los desarrollos y resultados obtenidos por las ciencias naturales ambientales. En particular si tiene sentido alguno desarrollar unas ciencias sociales que aborden la problemática ambiental de un modo desintegrado o si por el contrario ambos reinos del conocimiento deben estar informados y afectados mutuamente (Rosa, 1999; Stern, 1993; Stern et al., 1992).

(e) La oposición entre los que creen que los resultados, productos y metodologías de la ciencia social pueden usarse como medios para mejorar 
la participación y la democratización de la esfera pública, $-\mathrm{y}$ en particular, por lo que se respecta a las ciencias sociales ambientales, de las decisiones relativas a los problemas ambientales y de sostenibilidad-, y entre aquéllos que argumentan que tales propósitos no son posibles o apropiados (Renn et al., 1995; Dürrenberger et al., 1997; Tábara, 1998c y 1999a).

Todos ellos consisten en unos dilemas muy generales y amplios, casi imposibles de superar de manera mínimamente satisfactoria a medio plazo. Sin embargo, se tratan de unos retos epistemológicos y teóricos que afectan ineludiblemente a las cuestiones del trabajo de campo a las que se ha concentrado la atención de este artículo sobre qué medir, a quién medir, cómo medir y para qué medir la percepción ambiental.

Con respecto al qué medir, en este artículo se ha argumentado que la investigación en este campo se ha caracterizado por la existencia de dos ámbitos principales: el de la percepción de los problemas ambientales y sus interacciones sociales, por un lado, y el de los cambios de paradigmas culturales, por otro. En este aspecto cabe mencionar que la percepción de los problemas ambientales puede entenderse como una respuesta social que obedece a cambios reales que se producen en el medio físico y natural, o como un indicador de un cambio socialmente construido que se produce en el medio cultural de la sociedad. Así, se podría creer que aquellos sociólogos que parten de una perspectiva más social (construccionista) prefieren analizar ante todo el estudio de los cambios de paradigmas culturales para explicar luego las respuestas sociales ante estos fenómenos; o que los sociólogos realistas, estarían más dispuestos a analizar la percepción de los problemas ambientales dejando al margen si tales respuestas son o no fruto de cambios culturales más profundos. Sin embargo, no se produce ninguna correlación entre aquellos sociólogos que orientan su proceso de investigación y analizan resultados con base a presupuestos ecológico-realistas o sobre la base de planteamientos contruccionistas, dado que de hecho no existe oposición - aunque sí divergencia - entre el estudio empírico de los problemas ambientales y sus interacciones y el análisis de paradigmas, ni tampoco entre la posición realista y la posición construccionista en sociología. Tanto los unos como los otros pueden hacer uso de sondeos de opinión y pueden al mismo tiempo estudiar el cambio cultural y la percepción de los problemas ambientales desde una u otra perspectiva. El examen de la percepción social de los problemas ambientales, así como el análisis del cambio de paradigmas culturales, puede llevarse a cabo desde distintos enfoques epistemológicos y metodológicos, lo cual, claro está, producirá resultados e interpretaciones distintas.

En relación a la cuestión de a quién medir, la mayor parte de los estudios tanto sobre percepción ambiental, como sobre paradigmas culturales, se ha orientado a obtener datos relativos al conjunto de la población en general. Esto, en cierto modo, se corresponde con la preferencia cuantitativa que está presente en este campo de investigación, así como a un extendido supuesto que 
RIS

REIISTA INTERNACIONAL DE SOCIOLOGIA.

N" 28. Enero-Abril. 2001

cree posible estudiar actitudes, comportamientos o acciones de determinadas subpoblaciones a partir de muestras generales. No obstante, es obvio que las informaciones, contrastes e interpretaciones que se obtienen a partir de muestras específicamente obtenidas de grupos o subcolectivos concretos, son mucho más profundas y ricas que las recogidas a partir de muestras generales. Por este motivo, a pesar de que su aparente falta de representatividad sea motivo de crítica, su uso complementario con las segundas es altamente recomendable y en algunos casos imprescindible.

Respecto al cómo medir, puede decirse que la revisión de trabajos consultados muestra que esta línea de investigación tiene una clara preferencia metodológica por el uso de sondeos de carácter cuantitativo. A pesar de que en estos momentos se está produciendo una revitalización en el uso de metodologías cualitativas en este campo, éstas en ningún caso están sustituyendo a las cuantitativas ni parece haber signos de que ambas se triangulen suficientemente con el fin de producir unos análisis más densos de los resultados obtenidos. La superación de los escollos a los que se enfrenta la investigación de la percepción social del medio ambiente supone no sólo un cambio, ampliación o integración de los objetos de estudio, sino ante todo la necesidad de llevar a cabo una profunda transformación de los enfoques más tradicionales, de apostar por la innovación e imaginación metodológicas y de abrir algunas barreras disciplinares que pocos están dispuestos a atravesar. Creo que no es suficiente estudiar separadamente la percepción de los problemas ambientales o la evolución de los paradigmas culturales. Si verdaderamente queremos comprender las relaciones entre la sociedad y el medio ambiente, y las interacciones, representaciones y cuestiones que suscitan a los ciudadanos, es necesario incorporar la experiencia de más de veinte años de investigaciones en este campo, crear nuevos diseños metodológicos, y atreverse a elegir nuevos objetos de estudio aún por explorar. Todo ello, no tiene por qué suponer ningún "peligro" de pérdida de identidad o de distinción para nuestra subdisciplina, la sociología ambiental, o de incrementar la competencia por unos recursos de investigación escasos. Más bien lo contrario, puede rescatar a esta línea de investigación de una atonía harto peligrosa que, como alertaba Lowenthal en el caso de la geografia, puede acabar por paralizar su pleno desarrollo. Las aportaciones que la sociología puede hacer a la Evaluación Ambiental Integrada (EAI), por ejemplo, pueden contribuir a este objetivo, si bien esperemos que la EAI no erija de nuevo, como ya ocurrió en otros nuevos campos de investigación, sus propias fronteras disciplinares. En particular, en el presente artículo se ha defendido que si el objetivo de medir la percepción ambiental no se limita a realizar una descripción sociográfica de los problemas que se perciben sino que el investigador pretende ir algo más allá en la comprensión del significado y de las causas de los resultados obtenidos, la conexión entre teoría y práctica, y entre el conocimiento aportado por las ciencias naturales y el procedente de las ciencias sociales se hace imprescindible. 
Finalmente se ha manifestado en este artículo que la evaluación de la relevancia de los trabajos que pretenden medir la percepción ambiental puede realizarse con base a unos criterios puramente científicos o bien por su contribución a la posible resolución de problemas ambientales o de sostenibilidad específicos. En el primer caso, lo que puede interesar al investigador es poder demostrar el valor teórico añadido en la mejora de la calidad de sus interpretaciones por haber aplicado una aproximación empírica. En el segundo caso, los trabajos de campo orientados a la obtención de datos primarios, tanto cuantitativos como cualitativos, pueden utilizarse para intentar acercar determinadas preferencias y percepciones sociales a los procesos de decisión política en materia de medio ambiente. En este artículo se ha argumentado que tanto en un caso como en el otro tiene poco sentido, o ninguno, proceder con investigaciones empíricas separadas de la teoría o basadas únicamente en diseños cuantitativos o desintegrados. No obstante, la defensa de la integración no significa que las ventajas de llevar a cabo una investigación interdisciplinar en determinados campos y para cumplir determinadas funciones hagan irrelevante la investigación disciplinar. Sería ingenuo, y utópico a la vez, pensar que es posible disponer a medio plazo de una especie de metodología universal aplicable para estudiar todos los objetos de la realidad socioambiental. La sociología ambiental puede aprender, y mucho, de la investigación integrada e interdisciplinar, al igual que la sociología ambiental tiene mucho que ofrecer a las otras disciplinas que abordan las cuestiones de medio ambiente desde un enfoque social e integrado. Esta dirección (la teoría social y la sociología en particular) ofrece el ineludible potencial de síntesis y de análisis que es tan necesario, como ausente, en la investigación de problemáticas socioambientales en grandes poblaciones.

Muchas veces, los sondeos de opinión se utilizan para "probar" lo preocupados o poco preocupados que los ciudadanos están sobre el medio ambiente, $\mathrm{y}$, de este modo, poder legitimar o dar un apoyo más solvente a actuaciones públicas que, de otro modo, serían más dificiles de justificar. Sin embargo, el creciente número y la diversidad de actores que financian trabajos de este tipo, así como las mayores críticas a metodologías estándar faltas de innovación, de integración y de triangulación y que impiden la reflexión colectiva e informativamente densa de una temática tan compleja como la ambiental y de sostenibilidad, hace de esta línea de investigación un terreno cada vez más problemático y sujeto a la descontrucción. Por otro lado, es evidente que para probar que la población está preocupada o no por los problemas ambientales no es imprescindible el uso de sondeos de opinión, ni que esta técnica sea la única posible para tal propósito, aunque muy atractiva por lo mágico y poderoso, simbólicamente, que representa disponer de unos cuantos números con decimales. Y si por el otro, nuestro propósito no es tan político como académico, entonces la comprensión de las relaciones de la sociedad con el medio ambiente, objeto de la sociología ambiental, se puede beneficiar, y mucho, del uso de técnicas cuantitativas, pero 
en ningún caso debe limitarse a ellas.

En algunos países, como los Estados Unidos, el estudio de las percepciones públicas de los problemas del medio ambiente por parte de la sociología lleva realizándose desde la década de los setenta, mientras que, en Europa, la introducción de cuestiones ambientales en los sondeos de los principales centros de investigación social ha sido posterior y nunca ha superado el alcance de los trabajos estadounidenses. En España, los centros que han producido una mayor cantidad de datos sobre estas cuestiones han sido el Centro de Investigaciones Sociológicas (CIS) y el Centro para la Investigación de la Realidad Social (CIRES) aunque cada vez más están proliferando estudios llevados a cabo por otras instituciones públicas y privadas. La investigación española sobre percepción ambiental aun muestra un conjunto de debilidades que, por el momento, dificultan su pleno desarrollo. Entre estas dificultades se encuentra la fragmentación, la dificil comparabilidad, la falta de síntesis y flaqueza teórica, la irregularidad y la falta de triangulación metodológica, así como la ausencia de integración con otros ámbitos de acción y con los resultados de otras ciencias ambientales. No obstante, en España un conjunto de autores y de equipos diversos empiezan a avanzar decididamente, y a pesar de las dificultades de todo tipo con las que se enfrentan, hacia la progresiva consolidación de este área de investigación de trabajo.

\section{BIBLIOGRAFÍA}

ABOUD, A., A.J. SOFRANKO y S. NDIAYE (1996), "The Effects of Gender on Adoption of Conservation Practices by Heads of Farm Households in Kenya", Society and Natural Resources, 9: 447-463.

ADEOLA, F. O. (1998), “Cross-National Environmentalism Differentials: Empirical Evidence from Core and Noncore Nations", Society and Natural Resources, 11: 339-464.

ALEXANDER, J. y P. SMITH (1996), "Social Science and Salvation: Risk Society as Mythical Discourse", Zeitschrift für Soziologie, 25 (4): 251-262.

ALMENAR, R., E. BONO y E. GARCÍA (1998), "Percepciones, actitudes y valores de la población valenciana en relación a la sostenibilidad", en R. Almenar, E. Bono, E. García (coords.), La sostenibilidad del desarrollo: El caso valenciano, Valencia, Fundació Bancaixa.

ANDREWS, C.J. (1998), "Environmental Business Strategy: Corporate Leaders' Perceptions", Society and Natural Resources, 11: 531-540.

ARCURY, T. A. (1993), "Rural-Urban differences in Environmental Knowledge and Actions", Journal of Environmental Education, 25 (1):19-25.

BECK, U. (1992), Risk Society: Towards a New Modernity, Sage, Londres. 
BECHTEL, R.B., V.C. VERDUGO y J. de Q. PINHEIRO (1999), "Environmental Belief Systems: United States, Brazil, and Mexico", Journal of Cross Cultural Psychology, 30: 122-128.

BELL, A. (1994), "Climate and Opinion: Public and Media Discourse on the Global Environment", Discourse and Society, 5 (1): 33-64.

BERK, R.A. y D. SCHULMAN (1995), "Public Perceptions of Global Warming", Climatic Change, 29: 1-33.

BLAKE, D.E., N. GUPPY y P. URMETZER (1997), "Canadian Public Opinion and Environmental Action", Canadian Journal of Political Science, 30: 451-472.

BOSTROM, A., M.G. MORGAN, B. FISHHOFF y D. READ (1994), "What People Know about Global Climate Change?. 1. Mental Models", Risk Analysis, 14: 959-70.

BOYES, E. y M. STANISSTREET (1993), "The 'Greenhouse Effect': Children's perceptions of causes, consequences and cures", International Journal of Science Education, 15 (5): 531-552.

BURGESS, J., C. HARRISON y P. FILIUS (1995), Making the Abstract Real. A Cross-Cultural Study of Public Understanding of Global Environmental Change, Londres, University of College London, Dept. of Geography.

BURGESS, J., M. LIMB y C.M. HARRISON (1988a), "Exploring Environmental Values through the Medium of Small Groups. 1. Theory and Practice", Environmental Planning A., 20: 309-326

(1988b), "Exploring Environmental Values through the Medium of Small Groups2. Theory and Practice", Environmental Planning A., 20: 457-476.

BUTTEL, F.H. (1987), "New Directions of Environmental Sociology", Annual Review of Sociology, 13: 465-488.

BUTTEL, F.H. y W.L. FLINN (1976), "Economic Growth versus the Environment: Survey Evidence", Social Science Quarterly, 57: 410-20.

(1978), "The politics of Environmental Concern. The Impacts of Party Identification and Political Ideology on Environmental Attitudes", Environment and Behavior, 10 (1):17-36.

BUTTEL, F.H., O.B. MARTINSON y W.L. FLINN (1975), "Dimension and Hierarchy in the Perception of Environmental Problems", ponencia presentada en el "Pacific Chapter of the American Association for Public Opinion Research", Westwood, California.

BUTTEL, F. H. y P.J. TAYLOR (1992), "Environmental Sociology and Global Environmental Change: A Critical Assessment", Society and Natural Resources, 5: 211-230.

CARLSSON-KANYAMA, A., A. LINDEN y A. THELANDER (1999), "Gender Differences in Environmental Impacts From Patterns of Transportation -A Case Study from Sweden”, Society and Natural Resources, 12: 355-369. 


\section{RIS}

CARON, J.A. (1995), "The Black-White Environmental Concern Gap: An Examination of Environmental Paradigms", Journal of Environmental Education, 26 (2): 24-35.

CATTON, W.R. Jr. y R.E. DUNLAP (1978), "Environmental Sociology: A New Paradigm”, The American Sociologist, 13: 41-49.

(1980), "A New Ecological Paradigm for Post-Exuberant Sociology", American Behavioral Scientist, Vol. 24 (1): 15-47.

CENTRO DE INVESTIGACIONES SOCIOLÓGICAS (1996), "Ecología y medio ambiente", Datos de Opinión, 6 Agosto, Madrid, CIS.

(1997a), "Energia nuclear", Datos de Opinión, Octubre, Madrid, CIS.

(1997b), "La Ciencia avanza", Datos de Opinión, Marzo. Madrid, CIS.

(1999), "Del medio ambiente", Datos de Opinión, 21. Marzo, Madrid, CIS.

CIRES (1992 y 1994), "Medio ambiente", Madrid, CIRES.

CHAPMAN, G., K. KUMAR, C. FRASER y I. GABER (1997), Environmentalism and the Mass Media. The North-South Divide, Londres, Routledge.

CHULIÀ, E. (1995), "La conciencia medioambiental de los españoles en los noventa", ASP Research Paper, 12, Madrid.

COLLINS, T. (1997), "Environment gaining in polls", The Earth Times, 1-15 de agosto, p. 4.

COTGROVE, S. (1982), Catastrophe or Cornucopia: The Environment, Politics, and the Future, Nueva York, John Wiley.

(1980), "Environmentalism, Middle Class Radicalism and Politics", Sociological Review, 28 (2): 333-351.

CZECH, B. y KRAUSMAN (1999), "Public Opinion on Endangered Species Conservation and Policy”, Society and Natural Resources, 12: 469-479.

DE CASTRO, R. (2001), Comunicación electrónica interna dirigida a la Comisión Asesora del Ecobarómetro de Andalucía (05-04-01).

DÍAZ, M.A., A.E. RODRÍGUEZ y M.J. SALADO (1999), “Opinión Pública y Problemas Ambientales. El caso de las Instalaciones para el Tratamiento de Residuos en la Comunidad de Madrid", Revista Española de Investigaciones Sociológicas, 85: 251-275.

DOUGLAS, M. y A. WILDASKY (1982), Risk and Culture. An Essay on the Selection of Technical and Environmental Dangers, Berkeley, University of California Press.

DUNLAP, R.E. (1991a), "Trends in Public Opinion Toward Environmental Issues: 1965-1990". Society and Natural Resources, 4 (3): 285-312. 
(1991b), "Public Opinion in the 1980's. Clear Consensus Ambiguous Commitment", Environment, Vol. 33:10-15/32-37.

(1992), "The Health of the Planet Survey. A Preliminary Report on Attitudes Toward the Environment and Economic Growth Measured by surveys of Citizens in 22 nations to Date". Princeton, New Jersey: The George H. Gallup International Institute. Ponencia presentada en el Symposium on Current Developments in Environmental Sociology, Woudschoten, Zeist, Holanda.

(1993), "Of Global Concern. Results of the Health of the Planet Survey", Environment, Vol. 35(9): 7-39.

(1994), "International Attitudes towards Environment and Development", en H.O. Bergesen y G. Parmann (eds.), Green Globe Yearbook of International Co-operation on Environment and Development, Oxford University Press, pp. 115-126.

(1998), "Lay Perceptions of Global Risk. Public Views of Global Warming in Cross- National Context", International Sociology, Vol. 13(4): 473-498.

(2000), "Americans have a positive image of the environmental Movement", The Gallup Poll Monthly, 415 (abril): 19-25.

(2001), Comunicación personal realizada durante la celebración del Congreso New Natures, New cultures, New technologies, Fitzwillian College, Cambridge University, Reino Unido (julio).

DUNLAP, R.E. y W.R. Jr. CATTON (1983), "What Environmental Sociologists Have in Common (Whether Concerned With 'Built' or 'Natural' Environments)", Sociological Inquiry, 53: 113-135.

(1994a), "Struggling with Human Exemptionalism: The Rise, Decline and Revitalization of Environmental Sociology", American Sociologist, 25 (1): 5-29.

(1994b), "Toward an Ecological Sociology: The Development, Current Status, and Probable Future of Environmental Sociology", en W. D'Antonio, M. Sasaki y Y. Yonebayashi (eds.), Ecology, Society and the Quality of Social Life. New Brunswick (USA), Transaction Press.

DUNLAP, R.E., K.D. VAN LIERE, A. MERTIG, W.R. CATTON y R.E. HOWELL (1992), "Measuring Endorsement of an Ecological Worldview: a Revised NEP Scale'. Pullman: WA Washington State University, citado en G.Grenstadt (1999).

DUNLAP, R.E. y D.A. DILLMAN (1976), "Decline in Public Support for Environmental Protection: Evidence from a 1970-1974 panel study", Rural Sociology, 41: 382-390.

DUNLAP, R.E. y W.B. HEFFERNAN (1975), "Outdoor Recreation an Environmental Concern: An Empirical Examination”, Rural Sociology, 40: 18-30.

DUNLAP, R.E. y MERTIG (1994), "Global Environmental Concern: A Challenge to the PostMaterialism Thesis", Ponencia presentada al XIII Congreso Mundial de Sociologia, Bielefeld, Alemania. 
RIS

RETISTA INTERNACIONAL DE SOCIOLOGIA

N* 28. Enero-Abril, 2001

J. DAVID TÁBARA

DUNLAP, R.E. y R. SCARCE (1991), "Trends: Environmental Problems and Protection", Public Opinion Quarterly, 55(4):651-672.

DUNLAP, R.E. y K. VAN LIERE (1977), "Further Evidence of Declinining Public Concern with Environmental Problems: A Research Note", Western Sociological Review; 8: 108-112.

(1978), "The New Environmental Paradigm: A Proposed Measuring Instrument and Preliminary Results", Journal of Environmental Education, 9:10-19.

(1983), "Commitment to the Dominant Social Paradigm and Concern for Environmental Quality”, Social Science Quarterly, 65: 1013-1028.

DUNLAP, R.E, K.D. VAN LIERE y D.A. DILLMAN (1979), "Evidence of Decline in Public Concern with Environmental Quality: A Reply", Rural Sociology, 44 (1): 204-212.

DUNLAP, R.E, K.D. VAN LIERE y A.G. MERTIG (2000), "Measuring Endorsement of the New Ecological Paradigm: A Revised NEP Scale", Journal of Social Issues, 56. En prensa.

DÜRRENBERGER, G., J. BEHRINGER, U. DAHINDEN, A. GERGER, B. KASEMIR, C. QUEROI, R. SCHÜLE, D. TÁBARA, F. TOTH, M. VAN ASSELT, D. VASSILAROU, N. WILLI y C. JAEGER (1997), Focus Groups in Integrated Assessment. A manual for Participatory Research. Darmstadt University of Technology, Ulysses Working Paper 97-2.

EMPRESA NACIONAL DE RESIDUOS RADIOACTIVOS (1998), Actitudes de los españoles hacia el medio ambiente, Madrid, ENRESA-ASEP.

ESTER, P., S. SIMOES, M. AOYAGI-USUI y H. VINKEN (2000), The Global Environmental Survey: A Comparative Study of Cultural Change and Environmental Issues Among Mass Publics and Decision-Makers, Cambridge, The MIT Press (en prensa).

FUNTOWICZ, S.O. y J.R. RAVETZ (1991), "A New Scientific Methodology for Global Environmental Issues", en R. Constanza (ed.), Ecological Economics: The Science and Management of Sustainability, Nueva York, Columbia University Press.

FUNDACIÓN ENTORNO (2000), Hábitos de Consumo y Medio Ambiente en España, (Resumen) Madrid, Fundación Entorno.

GARCÍA FERRANDO, M. (1991), “Opinión pública y medio ambiente”, Sistema, 104-105:175-189.

GEISLER, C.C., O.B. MARTINSON y E.A. WILKENING (1977), "Outdoor Recreation and Environmental Concern", Rural Sociology, 42(2) 241-249.

GÓMEZ BENITO, C., F.J. NOYA y A. PANIAGUA (1999), "Actitudes y comportamientos hacia el medioambiente en España", Opiniones y Actitudes, n² 25, Madrid, CIS.

GRENDSTAD, G. (1999), "The New Ecological Paradigm Scale: Examination and Scale Analysis", Environmental Politics, 8(4):194-205. 
GRUNDY, S. (1998), 'You Cannae Stop the Future'. Young People and Environmental Issues, Tesis doctoral presentada en la Universidad de Edimburgo, Mayo.

HANNIGAN, J. (1995), Enironmental Sociology. A Social Constructionist Approach, London y Nueva York, Routledge.

HARRIS/UNEP (1988), Public and Leadership Attitudes to the Emironment in Four Continents. Nueva York, Louis Harris \& Associates, UNEP.

HILLCOAT, J., K. FORGE, J. FIEN y E. BAKER (1995), "I Think It's Really Great that Someone is listening to Us...' : Young People and the Environment", Environmental Education Research,1(2): 159-171.

HOFFMAN, A.J. (1996), "Trends in Corporate Environmentalism. The Chemical and Petroleum Industries, 1960-1993", Society and Natural Resources, 9:47-64.

HONNOLD, J.A. y L.D. NELSON (1979), "Support for Resource Conservation: A prediction Model”, Social problems, Vol. 27(2):220-234.

INGLEHART, R. (1977), The Silent Revolution. Changing Values and Political Styles among Western Publics, Princeton, Princeton University Press.

(1990), Culture Shift in Advanced Industrial Society, Princeton, Princeton University Press.

(1995), "Public Support for Environmental Protection: Objective Problems and Subjective Values in 43 Societies", Political Science and Politics, Vol, XXVIII no.1.

INSTITUT DE CIÈNCIES POLÍTIQUES I SOCIALS (1991-1998), Sondeig d'Opinió Pública a Catalunya, Barcelona, ICPS.

INSTITUTO IDES (1986), "Estudio sociológico sobre medio ambiente en España", Información Ambiental, 9:I-X.

INSTITUTO DE ESTUDIOS SOCIALES AVANZADOS DE ANDALUCÍA (1996), Percepción de los Problemas Medioambientales por la Población Andaluza. Informe de sintesis, Córdoba, IESA-Andalucía, Marzo.

JAEGER, C.C., G. DÜRRENBERGER, B. KASTENHOLZ y B. TRUFFER (1993), "Determinants of Environmental Action with Regard to Climatic Change", Climatic Change, 23:193-221.

JAEGER, C.C., R. SCHÜLE y B. KASEMIR (1999), "Focus Groups in Integrated Assessment: A Micro-cosmos for Reflexive Modernization", Innovation, 12(2):195-219.

JASANOFF, S. y B. WYNNE (1998), "Science and decisionmaking", en S. Rayner y E. Malone (eds.), Human Choice and Climate Change. Vol.1. Columbus, Ohio, Battelle Press.

JONES, R.E. (1996), "Black Concern for the Environment: Myth Versus Reality", Society and Natural Resources, 11:209-229. 
RIS

RETISTA INTERNACIONAL DE SOCIOLOGI:

N" 28. Enero-Abril. 2001

J. DATIDTIBALR

JONES, R.E. y R.E. DUNLAP (1992), "The Social Bases of Environmental Concern: Have They Changed Over Time?", Rural Sociology, 57(1):28-47.

KASEMIR, B., U. DAHINDEN, A. GERGER SWARTLING, R. SCHÜLE, D. TÁBARA y C.C. JAEGER (2000), "Citizens' perspectives on Climate Change and Energy Use", Global Environmental Change, 10(3):169-184.

KEMPTON, W., J.S. BOSTER y HARTLEY (1995), Environmental Values in American Culture, Cambrige, MA, The MIT Press.

LÖFSTEDT, R.E. (1995), "Why are Public Perception Studies on the Environment Ignored?", Global Environmental Change, 5(2):83-85.

LOWE, G.D. y T.K. PINHEY (1982), "Rural-Urban Differences in Support for Environmental Protection", Rural Sociology, 47 (1):114-128.

LOWENTHAL, D. (1987), "Environmental Perception: An Odyssey of Ideas", Journal of Environmental Psychology, 7:337-346.

MACNAGHTEN, P. W., R. GROVE-WhYTE, M. JACOBS y B. WYNNE (1995), Public Perceptions and Sustainability in Lancashire. Indicators Insitutions, Participation, Lancaster, Lancaster University, Centre for the Study of Environmental Change.

MARKETING SYSTEMS (1996), Principals Resultats de l'Estudi de l'Index de Conscienciació Ambiental de Catalunya, Generalitat de Catalunya, Direcció General de Promoció i Educació Ambiental del Departament de Medi Ambient, Barcelona, Dept. de Medi Ambient.

MARTÍN, T. (1999), "La percepción social del medioambiente en España. Perspectivas del movimiento ecologista", en M. Pardo, Sociología y Medio Ambiente. Estado de la Cuestión. Pamplona, Fundación Fernando de los Ríos, Universidad Pública de Navarra y Foro Formación Ediciones.

MERTIG, A. y R. DUNLAP (1995), "Public Aproval of Environmental Protection and other Social Movement goals in Western Europe and the United States", International Journal of Public Opinion Research, 7(2):145-156.

MILBRATH, L.W. (1984), Environmentalists, Vanguard for a New Society, Nueva York, State University of New York Press.

MOHAI, P. (1992), "Men, Women and the Environment: An Examination of the Gender Gap in Environmental Concern and Activism", Society and Natural Resources, 5:1-19.

(1985), "Public Concern and Elite Involvement in Environmental Conservation Issues", Social Science Quarterly, :820-837.

MORRISON, D. E. y R.E. DUNLAP (1986), "Environmentalism and Elitism: A conceptual and Empirical Analysis", Environmental Management, 10:581-589.

NAVARRO YÁÑEZ, C.J. (1998), "Conciencia ambiental y perfil social del ambientalismo. Una cuestión de competencia política", Revista Internacional de Sociologia, 19-20:69-101. 
(2000), "Competencia politica, ambientalismo y cambio social. Normas y comportamientos ambientales en Andalucia", Politica y Sociedad (en prensa).

NOE, F.P. y R. SNOW (1989-90), "Hispanic Cultural Influence on Environmental Concern”, Jouinal of Environmental Education, 21: 27-34.

OECD (1991), OECD Environmental Data 1991, Paris, OECD.

OLSEN, M.E., D.G. LODWICK y R.E. DUNLAP (1992), Viewing the World Ecologically, Boulder, Westview.

OSTMAN, R.E. y J.L. PARKER (1986-7), “A Public's Environmental Information Sources and Evaluations of Mass Media", Journal of Environmental Education: 18(2):9-17.

OZANE, L.K., C.R. HUMPHREY y P.M. SMITH (1999), "Gender, Environmentalism, and Interest in Forest Certification: Mohai's Paradox Revisited", Society and Natural Resources, 12: 613-622.

READ, D., A. BOSTROM, M.G. MORGAN, B. FISCHHOFF y T. SMUTS (1994), "What People Know about Global Climate Change?. 2. Surveys Studies of Educated People", Risk Analysis, 14: 971-958.

RENN, O. et al. (eds.) (1995), Fairness and Competence in Citizen Participation. Evaluating Models for Environmental Discourse, Kluwer, Academic Publishers.

ROSA, E.A. (1999), "The quest to Understand Society and Nature: Looking Back, but Mostly Forward", Society and Natural Resources, 12: 371-376.

ROSA, E.A. y R.E. DUNLAP (1994), "Nuclear Power: Three Decades of Public Opinion", Public Opinion Quarterly, 58: 295-325.

SKRENTNY, J. (1993), "Concern for the Environment: A Cross-National Perspective", International Journal of Public Opinion Research, 5(4):334.-352

STERN, P.C. 1993), "A Second Environmental Science: Human-environment interactions", Science, 260:1897-1899.

STERN, P.C., O.R. YOUNG y D. DRUCKMAN (eds.) (1992), Global Environmental Change. Understanding the Human Dimensions, Washington, D.C., National Academy Press.

TÁBARA, D. (1993), “Mitjans de Comunicació i Medi Ambient”, Perspectiva Social, 81-95.

(1995), Percepció Pública i Acció Social en Problemes de Medi Ambient, Tesis doctoral, Barcelona, Universidad de Barcelona, Departament de Sociologia i Metodologia de les Ciències Socials.

(1996), La Percepció dels Problemes de Medi Ambient. Barcelona: Ed. Beta \& Departament de Medi Ambient de la Generalitat de Cataluña.

(1997), "La Percepción de la Degradación Ambiental”, publicado en el monográfico sobre medio 


\section{R I S}

ambiente, Winempresa (junio): 71-76.

(1998a), "La percepció social del medi ambient. El cas de Menorca", en B. Tomàs Vives (Coord.). El Medi Ambient a les Illes Balears. Qui es qui?. Papers de Medi Ambient, $\mathrm{n}^{\circ} 6$, pp. 158-170.

(1998b), "Towards Integration of Mass Media Environmental Communication in Europe", Contribution to an Expert Corner Report. European Environmental Agency \& Centre d'Estudis d'Informació Ambiental. Barcelona: ICT-CEIA.

(1998c) "Citizen participation and equity in global environmental change: the ia-focus group process", Ponencia presentada a la cuarta sesión de encuentro Lifestyles and EnvironmentParticipation and Equity, Bruselas, Comisión Europea, 16-17 Marzo.

(1999), Acció ambiental. Aprenentage i participació vers la sostenibilitat. Binissalem, Illes Balears, Di7 Edicions \& Associació Catalana d'Educació Ambiental-Associació Balear d'Educació Ambiental.

(2000), "Parlem del clima: aportació de la sociologia qualitativa a l'Avaluació Integrada del canvi climàtic", Revista Catalana de Sociologia, 13:45-76.

(2001a), "Participació i coneixement per a la sostenibilitat", Societat i Sostenibilitat, Caixa de Balears-Sa Nostra, Palma de Mallorca, Islas Baleares: Fundació Sa Nostra, Papers de Medi Ambient, 12, pp. 95-106.

(2001b), "Participación cualitativa y evaluación Integrada del Medio Ambiente. Aspectos metodológicos en cuatro estudios de caso", Documents d'Anàlisi Geogràfica (en prensa).

TÁBARA, D. y C. QUEROL (1999), "Evaluación Integrada del cambio climático: experiencias de grupos de discusión en el Área Metropolitana de Barcelona", en M. Pardo (coord.), Sociología y Medio Ambiente. Estado de la Cuestión. Pamplona, Fundación Fernando de los Ríos, Universidad Pública de Navarra \& Foro Formación Ediciones.

TÁBARA, D., J. VAN DER SLUIJS y W. TUNINSTRA (2000), "Ideal Types, Uncertainty and Integrated Environmental Assessment", Uncertainty and Integrated Environmental Assessment, European Forum for Integrated Environmental Assessment, Viena, EFIEA. Documento de trabajo interno.

TILLOCK, H. y D.E. MORRISON (1979), "Group Size and Contributions to Collective Action: An Examination of Olson Theory Using Data From Zero Population Growth", en L. Kriesberg (ed.), Research in Social Movements, Conflicts and Change, Nueva York, JAI Press.

TREMBLAY, K. y R.E. DUNLAP (1978), "Rural-Urban Residence and Concern with Environmental Quality: A Replication and Extension", Rural Sociology, 43(3): 474-491.

VAN LIERE, K.D. (1981), “Environmental Concern. Does It Make a Difference How It's Measured?", Environment and Behavior, 13 (6):651-675.

VAN LIERE, K.D. y P.F. NOE (1981), "Outdoor Recreation and Environmental Attitudes: Further Examination of the Dunlap-Heffernan Thesis", Rural Sociology, 46(3):505-513. 
VAN LIERE, K.D. y R.E. DUNLAP (1980), "The Social Bases of Environmental Concern: A Review of Hypotheses, Explanations, and Empirical Evidence", Public Opinion Quarterly, 44:181-197.

VAN LIERE, K.D. y F.P. NOE (1981), "Outdoor Recreation and Environmental Attitudes: Further Examination of the Dunlap-Heffernan Thesis", Rural Sociology, 46(3):505-513.

VAUGHMAN, E. y B. NORDERNSTAM (1991), "The Perception of Environmental Risks Among Ethnically Diverse Groups", Journal of Cross-cultural Psychology, 22(1):29-60.

WEBER. R. y M. CORRADO (1993), "International Attittudes to the Environment", Londres, Mori Social Research Institute \& World Wide Fund for Nature. Ponencia presentada para la WAPOR Conference, Copenhague.

WHITE, A.V.T. (1977), Guidelines for Field Studies in Environmental Perception, International Coordinating Council of the Programme on Man and the Biophere, MAB Technical Notes, $\mathrm{n}^{\circ}$ 5, París, UNESCO.

WYNNE, B. (1992), "Uncertainty and Environmental Learning: Reconceiving Science and Policy in the Preventive Paradigm", Global Environmental Change, 2(2):11-127.

(1994), "Scientific Knowledge and the Global Environment", en M. Redclift y T. Benton (eds.), Social Theory and the Global Environment, London, Routledge.

WORCESTER, R. (1993), "Public and Elite Attitudes to the Environment", International Journal of Public Opinion Research, 5(4): 315-334.

(1994), "Societal Values, Behaviour and Attitudes in Relation to the Human Dimensions of Global Environmental Change: Use of an Environmental Activist Scale", Ponencia presentada en el XVI IPSA World Congress, Berlín, Agosto.

(1996), "Not So Green as Cabbage Looking': Comparing Environmental Activism of the Public and of Journalists", ponencia presentada en la Conferencia "Reporting the Environment: Capitalism Goes Green", Centre for Journalism Studies, University of Wales, Cardiff, Gran Bretaña. 